\title{
Treatment of the polar coordinate singularity in axisymmetric wave propagation using high-order summation-by-parts operators on a staggered grid
}

\author{
Bo Prochnow ${ }^{\mathrm{a}}$, Ossian O’Reilly ${ }^{\mathrm{a}, \mathrm{b}, *}$, Eric M. Dunham ${ }^{\mathrm{a}, \mathrm{c}}$, N. Anders Petersson ${ }^{\mathrm{d}}$ \\ ${ }^{a}$ Department of Geophysics, Stanford University, Stanford, CA, USA \\ ${ }^{b}$ Department of Mathematics, Division of Computational Mathematics Linköping \\ University, SE-581 83 Linköping, Sweden \\ ${ }^{c}$ Institute for Computational and Mathematical Engineering, Stanford University, Stanford, \\ $C A, U S A$ \\ ${ }^{d}$ Center for Applied Scientific Computing, Lawrence Livermore National Laboratory, PO \\ Box 808, Livermore, CA 94551, United States
}

\begin{abstract}
We develop a high-order finite difference scheme for axisymmetric wave propagation in a cylindrical conduit filled with a viscous fluid. The scheme is provably stable, and overcomes the difficulty of the polar coordinate singularity in the radial component of the diffusion operator. The finite difference approximation satisfies the principle of summation-by-parts (SBP), which is used to establish stability using the energy method. To treat the coordinate singularity without losing the SBP property of the scheme, a staggered grid is introduced and quadrature rules with weights set to zero at the endpoints are considered. The accuracy of the scheme is studied both for a model problem with periodic boundary conditions at the ends of the conduit and its practical utility is demonstrated by modeling acoustic-gravity waves in a magmatic conduit.
\end{abstract}

Keywords: polar coordinate singularity, summation-by-parts, high-order finite difference method, staggered grid, axisymmetric wave propagation

\footnotetext{
* Corresponding author

Email addresses: bprochnow@alumni.stanford.edu (Bo Prochnow), ooreilly@stanford.edu (Ossian O'Reilly), edunham@stanford.edu (Eric M. Dunham), petersson1@llnl.gov (N. Anders Petersson)
}

Preprint submitted to Computers \&S Fluids

February 6, 2017

(C) 2017. This manuscript version is made available under the Elsevier user license http://www.elsevier.com/open-access/userlicense/1.0/ 


\section{Introduction}

Wave propagation in fluid-filled tubes or conduits arises in many systems, ranging from pulsatile flow in blood vessels $[1,2]$ to acoustic-gravity waves in magmatic conduits beneath volcanoes [3]. Fluid viscosity, a key source of dissipation, introduces diffusion terms to the governing equations. When expressed in cylindrical coordinates, the radial component of the diffusion operator (i.e., the Laplacian) takes the form

$$
\frac{1}{r} \frac{\partial}{\partial r}\left(r \frac{\partial v}{\partial r}\right)
$$

for velocity field $v$ along the axis of the conduit. Finite difference discretization of this operator must contend with the coordinate singularity at $r=0$, the center of the conduit.

There have been several approaches to the treatment of the $1 / r$ coordinate singularity using finite difference methods. These methods are not necessarily limited to axisymmetric problems. As summarized in $[4,5]$, the main approaches to treating this pole have been to use analytic techniques or choose a specific discretization to treat the singularity, or to transform the domain or governing equations to avoid imposing a boundary condition at the pole. The former approach is used in $[6,7,8]$, which impose l'Hôpital's rule near the pole to write any $1 / r$ terms in a form that is nonsingular near $r=0$. Mohseni and Colonius [9] avoid the need for a special boundary closure (and the corresponding potential for reduced boundary accuracy) by remapping the computational domain such that no grid point is placed on the singularity. In [10], the incompressible Navier-Stokes equations in cylindrical coordinates are solved on a staggered grid. Terms like (1.1) are never evaluated at $r=0$; similar terms with coordinate singularities involving the radial flux are handled by using $r$ times the radial flux as a dependent variable instead of the radial flux.

Summation-by-parts (SBP) finite difference methods, first introduced in [11] and summarized in $[12,13]$, are well-suited to modeling wave propagation in fluid-filled conduits since they allow for the construction of higher-order spatial 
discretizations for which the system energy rate can be computed and used to establish time-stability. While we focus on a system with periodic boundary conditions on the top and bottom of the conduit, more general boundary conditions can be weakly enforced using the simultaneous-approximation-term (SAT) technique $[14,13,15]$. The implementation of SBP methods for wave propagation in cylindrical coordinates requires finding a way to treat the $1 / r$ singularity while preserving the SBP properties of the operators. In [16], a second order accurate discretization is constructed by using the axisymmetry of the system to impose a regularity condition at $r=0$. This approach uses standard second order centered difference operators (modified to satisfy the regularity condition), but is not directly generalizable to higher orders of accuracy. A related method is used in [17] to construct SBP operators that are second- and fourth-order accurate on interior points by manipulating the stencil of standard difference operators near the boundary using regularity conditions and l'Hôpital's rule to eliminate singular terms.

Here we present a novel discretization scheme utilizing SBP finite difference operators on a staggered grid. This approach permits construction of high-orderaccurate operators with proper boundary treatment, thereby allowing stability proofs via energy estimates. We present these operators first in the 1-D (radial) context, then generalize to the 2-D problem of axisymmetric, long-wavelength waves propagating in a cylindrical conduit filled with a viscous fluid. This problem features narrow boundary layers near the conduit walls when the viscosity is sufficiently small. After establishing stability and accuracy of the method, we present results regarding optimal selection of grid spacings in the radial and axial directions based on consideration of the relative size of the wavelength and boundary layer thickness. We close by demonstrating the utility of these new operators for the more complex problem of acoustic-gravity waves in magmatic conduits. While the operators presented here are specifically designed for the coordinate singularity problem for the radial component of the Laplacian operator in cylindrical coordinates, it is likely that the approach could be extended to related problems in spherical coordinates, non-axisymmetric problems, or other 
coordinate systems having coordinate singularities.

\section{Continuous formulation of the model problem}

\subsection{Governing equations}

We consider wave propagation and diffusion in a cylindrical conduit. The fluid pressure $p=p(t, z)$ and the axial component of the velocity field $v=$ $v(t, z, r)$ are treated as being axisymmetric (no azimuthal dependence), where $r$ and $z$ are the dimensionless radial and axial coordinates, respectively. We define a two-dimensional rectangular domain $\Omega$ as $0 \leq r \leq 1$ and $0 \leq z \leq 2 \pi$, where $r=0$ is at the center of the conduit and $r=1$ is at the wall, while $z=0$ is the conduit bottom and $z=2 \pi$ is the top. By restricting attention to wavelengths much greater than the conduit radius, the radial momentum balance establishes, to a good approximation, the uniformity of pressure in the radial direction [1]. In this limit, a dimensionless and linearized statement of conservation of mass, together with linearized relations between density perturbations and pressure (and possibly also perturbations in cross-sectional area and local pressure), becomes

$$
\frac{\partial p}{\partial t}+\frac{\partial u}{\partial z}=0
$$

where

$$
u(t, z)=2 \int_{0}^{1} v(t, z, r) r d r
$$

is the cross-sectionally averaged fluid velocity. The factor of 2 is included such that if $v$ is constant the cross-sectionally averaged velocity $u$ will equal $v$ exactly.

The linearized, dimensionless statement of conservation of momentum includes both a pressure gradient term and a viscous term from shearing on axisymmetric surfaces of constant $r$ :

$$
\frac{\partial v}{\partial t}+\frac{\partial p}{\partial z}=\epsilon \frac{1}{r} \frac{\partial}{\partial r}\left(r \frac{\partial v}{\partial r}\right)
$$

Additional viscous terms in the linearized Navier-Stokes equation are negligible in comparison in the long wavelength limit of interest. The dimensionless 
parameter $\epsilon$ is defined in terms of the fluid viscosity $\mu$, density $\rho$, and conduit radius $R$ as

$$
\epsilon=\frac{\mu}{\rho R^{2} \omega},
$$

where $\omega=c k$ is the characteristic angular frequency, defined in terms of the acoustic wave speed $c$ and the axial wavenumber $k$. For small values of $\epsilon$, narrow boundary layers develop near the conduit walls, where a no-slip boundary condition is imposed:

$$
v(t, z, r=1)=0 .
$$

To make the presentation more concise, we consider periodic boundary conditions in the axial direction:

$$
v(t, z=2 \pi, r)=v(t, z=0, r), \quad p(t, z=2 \pi)=p(t, z=0) .
$$

\subsection{Energy balance}

In this section, we derive an energy estimate for the problem. In cylindrical coordinates, we define the continuous $L_{2}$-norm for a real-valued function $\psi(t, z, r)$ as $\|\psi\|^{2}=\int_{0}^{2 \pi} \int_{0}^{1} \psi^{2} r d r d z$, and define the energy

$$
E(t)=\frac{1}{2}\|v\|^{2}+\frac{1}{2}\|p\|^{2}=\frac{1}{2} \int_{0}^{2 \pi} \int_{0}^{1}\left(v^{2}+p^{2}\right) r d r d z .
$$

The first term is the kinetic energy of the fluid, while the second term is the potential energy stored through compression/expansion of the fluid and/or elastic deformation of the conduit walls.

Prior to the imposition of boundary conditions, the rate of change of energy is

$$
\frac{d E}{d t}=-\frac{1}{2}[u p]_{z=0}^{2 \pi}+\epsilon \int_{0}^{2 \pi}\left[v r \frac{\partial v}{\partial r}\right]_{r=0}^{1} d z-\epsilon \int_{0}^{2 \pi} \int_{0}^{1}\left(\frac{\partial v}{\partial r}\right)^{2} r d r d z .
$$

The first term in this expression, which corresponds to the work done by external forces at the top and bottom of the conduit ( $z=2 \pi$ and $z=0$ ), equals zero when the periodic boundary condition (2.6) is imposed. The second term represents 
the change in energy due to external forces at the walls of the conduit $(r=1)$. When the no-slip boundary condition (2.5) is imposed, this term is zero at $r=1$. With no sources or sinks of mass or singular forces along the axis, $v$ and $\partial v / \partial r$ are bounded at $r=0$, so the term is zero at $r=0$ as well. The energy rate thus reduces to

$$
\frac{d E}{d t}=-\epsilon\left\|\frac{\partial v}{\partial r}\right\|^{2}
$$

The right hand side of this equation represents the rate of energy loss due to viscous dissipation in the fluid. Because $\epsilon \geq 0, d E / d t \leq 0$.

\section{Finite difference approximation of the radial diffusion operator}

The construction of a provably stable numerical scheme requires special treatment of the coordinate singularity at $r=0$. To overcome this challenge, we introduce a shifted grid with interior points offset by $\Delta r / 2$ relative to an equidistant grid with grid spacing $\Delta r$. As shown in section 2.2, no boundary condition should be specified at $r=0$. We proceed by constructing summation-by-parts operators that also possess this property.

\subsection{Definitions}

We define two grids in the radial direction: $\mathbf{r}_{+}$, which is an equidistant grid with grid spacing $\Delta r=1 / N$, and $\mathbf{r}_{-}$, which is staggered relative to $\mathbf{r}_{+}$(see Figure 1). We have

$$
\mathbf{r}_{+}=\left[\begin{array}{llll}
r_{0} & r_{1} & \ldots & r_{N}
\end{array}\right]^{T} \in \mathbb{R}^{N+1}, \quad \mathbf{r}_{-}=\left[\begin{array}{lllll}
r_{0} & r_{1 / 2} & r_{3 / 2} & \ldots & r_{N-1 / 2} \\
r_{N}
\end{array}\right]^{T} \in \mathbb{R}^{N+2},
$$

where

$$
r_{j}=j \Delta r, \text { for } j \in[0, N], \quad r_{j+1 / 2}=\left(j+\frac{1}{2}\right) \Delta r, \text { for } j \in[0, N-1] .
$$

Note that the endpoints of both grids coincide and that the grid point $r_{0}$ is placed at $r=0$. 


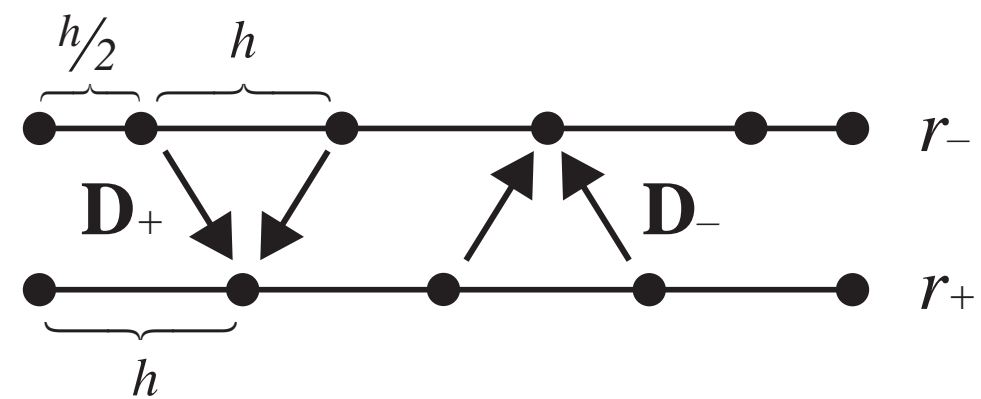

Figure 1: The $\mathbf{r}_{-}$and $\mathbf{r}_{+}$grids used in the discretization. The difference operator $\mathbf{D}_{+}$acts on a grid function on the $\mathbf{r}_{-}$grid and approximates the first derivative on the $\mathbf{r}_{+}$grid. The difference operator $\mathbf{D}_{-}$is defined in a similar manner.

We introduce grid functions $\phi_{+}$and $\phi_{-}$that represent a continuous function $\phi(r)$ on the grids $\mathbf{r}_{+}$and $\mathbf{r}_{-}$, respectively. Thus,

$$
\phi_{+}=\left[\begin{array}{llll}
\phi_{0} & \phi_{1} & \ldots & \phi_{N}
\end{array}\right]^{T}, \phi_{-}=\left[\begin{array}{llll}
\phi_{0} & \phi_{1 / 2} & \ldots & \phi_{N-1 / 2}
\end{array} \phi_{N}\right]^{T} .
$$

We define the difference operator $\mathbf{D}_{+}$to act on a grid function $\phi_{-}$and approximate the first derivative on the $\mathbf{r}_{+}$grid. Similarly, we define the difference operator $\mathbf{D}_{-}$to act on a grid function $\phi_{+}$and approximate the derivative on the $\mathbf{r}_{-}$grid. Figure 1 illustrates an example with second order accurate difference operators,

$$
\begin{gathered}
\left.\frac{\partial \phi}{\partial r}\right|_{r_{j}} \approx\left(\mathbf{D}_{+}^{(2)} \phi_{-}\right)_{j}=\frac{\phi_{j+1 / 2}-\phi_{j-1 / 2}}{\Delta r}, \\
\left.\frac{\partial \phi}{\partial r}\right|_{r_{j-1 / 2}} \approx\left(\mathbf{D}_{-}^{(2)} \phi_{+}\right)_{j-1 / 2}=\frac{\phi_{j}-\phi_{j-1}}{\Delta r} .
\end{gathered}
$$

Difference operators that satisfy the principle of summation-by-parts are defined by combining central difference approximations in the interior of the domain and one-sided difference approximations near the boundary.

Definition 1. The pair of difference operators $\mathbf{D}_{+} \in \mathbb{R}^{(N+1) \times(N+2)}$ and $\mathbf{D}_{-} \in$ $\mathbb{R}^{(N+2) \times(N+1)}$ are first derivative staggered grid summation-by-parts operators if

1. The difference operators $\mathbf{D}_{+}$and $\mathbf{D}_{-}$are $2 p$ th order accurate in the interior and at least pth order accurate near the boundary. 
2. There exists (semi-)definite diagonal matrices $\mathbf{P}_{+}$and $\mathbf{P}_{-}$that define the discrete scalar products,

$$
\begin{gathered}
\boldsymbol{\psi}_{+}^{T} \mathbf{P}_{+} \boldsymbol{\phi}_{+}:=\left(\boldsymbol{\psi}_{+}, \boldsymbol{\phi}_{+}\right)_{+}=\Delta r \sum_{j=0}^{N} w_{j} \psi_{j} \phi_{j}, \\
\boldsymbol{\psi}_{-}^{T} \mathbf{P}_{-} \boldsymbol{\phi}_{-}:=\left(\boldsymbol{\psi}_{-}, \boldsymbol{\phi}_{-}\right)_{-}=\Delta r \sum_{j=0}^{N-1} \tilde{w}_{j+1 / 2} \psi_{j+1 / 2} \phi_{j+1 / 2},
\end{gathered}
$$

with weights $w_{j}>0, \tilde{w}_{j+1 / 2}>0$, and corresponding (semi-)norms $\left\|\phi_{+}\right\|_{+}^{2}=$ $\left(\phi_{+}, \phi_{+}\right)_{+}$and $\left\|\phi_{-}\right\|_{-}^{2}=\left(\phi_{-}, \phi_{-}\right)_{-}$.

3. The first and last diagonal elements of $\mathbf{P}_{-}$are zero.

4. The difference operators satisfy the summation-by-parts property

$$
\left(\boldsymbol{\psi}_{+}, \mathbf{D}_{+} \boldsymbol{\phi}_{-}\right)_{+}+\left(\mathbf{D}_{-} \boldsymbol{\psi}_{+}, \boldsymbol{\phi}_{-}\right)_{-}=\psi_{N} \phi_{N}-\psi_{0} \phi_{0},
$$

for all real-valued grid functions $\boldsymbol{\psi}_{+}$and $\boldsymbol{\phi}_{-}$. This property can be expressed in matrix form as

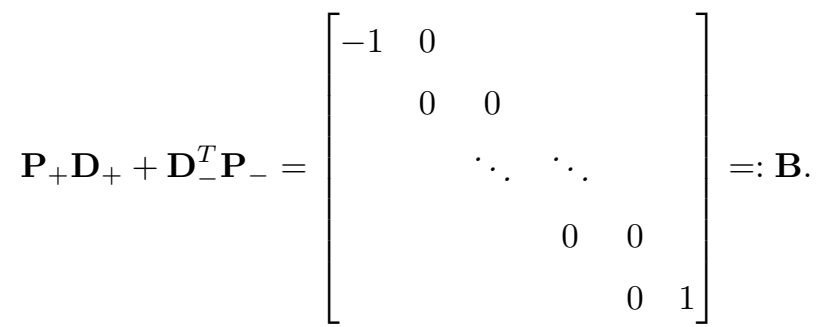

Remark 1. By construction, the scalar products (3.2) and (3.3) represent quadrature rules and their order of accuracy is addressed in Appendix A.

Remark 2. The requirement that the first and last diagonal elements of $\mathbf{P}_{-}$ are zero is essential for the treatment of the polar coordinate singularity. This implies that the first and last rows of $\mathbf{D}_{-}$are are only determined by the accuracy constraint (1) of Definition 1 and can be chosen independently of all other rows in $\mathbf{D}_{-}$.

As an example, we show a pair of finite difference operators that satisfy the 
conditions of Definition 1 for $p=1$ :

$$
\begin{gathered}
\mathbf{D}_{+}=\frac{1}{\Delta r}\left[\begin{array}{cccccc}
-2 & 2 & & & & \\
& -1 & 1 & & & \\
& & \ddots & & \\
& & -1 & 1 & \\
& & & -2 & 2
\end{array}\right], \\
\mathbf{D}_{-}=\frac{1}{\Delta r}\left[\begin{array}{cccc}
-1 & 1 & & \\
-1 & 1 & & \\
& \ddots & \ddots & \\
& & -1 & 1 \\
& & -1 & 1
\end{array}\right] .
\end{gathered}
$$

The corresponding weights in the scalar products are $\mathbf{P}_{+}=\Delta r \operatorname{diag}(1 / 2,1, \ldots, 1,1 / 2)$ and $\mathbf{P}_{-}=\Delta r \operatorname{diag}(0,1, \ldots, 1,0)$.

\subsection{Construction of higher order staggered grid SBP operators}

Standard SBP approximations of first derivatives are commonly defined by $\mathbf{D}=\mathbf{P}^{-1} \mathbf{Q}$, where $\mathbf{Q}+\mathbf{Q}^{T}=\operatorname{diag}(-1,0, \ldots, 0,1)$. Unfortunately, this approach does not generalize to the staggered case because the matrix $\mathbf{P}_{-}$is singular, necessitating a different approach.

Let $m(p) \geq 2 p$ be an integer, which equals the number of modified difference stencils near each boundary. The starting point of our construction is to choose a compact interior difference stencil of order $2 p$. In the regular grid, the interior stencil will be used at the points $r_{j}$, for $j \in[m, N-m]$. In the staggered grid, the interior stencil will be used at $r_{j-1 / 2}$, for $j \in[m, N-m+1]$. At all interior points, the weights in the scalar products are taken to be to $w_{j}=1$ and $\tilde{w}_{j-1 / 2}=1$.

Let $k \geq 0$ be an integer and let the grid functions $\mathbf{r}_{+}^{k}$ and $\mathbf{r}_{-}^{k}$, be discretizations of the monomial function $f(r)=r^{k}$, i.e., $\mathbf{r}_{+}^{k}=\left[r_{0}^{k}, r_{1}^{k}, \ldots, r_{N}^{k}\right]^{T}$ and $\mathbf{r}_{-}^{k}=\left[r_{0}^{k}, r_{1 / 2}^{k}, \ldots, r_{N-1 / 2}^{k}, r_{N}^{k}\right]^{T}$. We define a pair of staggered grid SBP 
operators to have $p$ th order boundary accuracy if they satisfy

$$
\begin{aligned}
& \mathbf{Q}_{+} \mathbf{r}_{-}^{k}=k \mathbf{P}_{+} \mathbf{r}_{+}^{k-1} \\
& \mathbf{Q}_{-} \mathbf{r}_{+}^{k}=k \mathbf{P}_{-} \mathbf{r}_{-}^{k-1}
\end{aligned}
$$

for $k=0,1, \ldots, p$. Here, $\mathbf{Q}_{+}=\mathbf{P}_{+} \mathbf{D}_{+}$and $\mathbf{Q}_{-}=\mathbf{P}_{-} \mathbf{D}_{-}$. Note that the first condition is equivalent to $\mathbf{D}_{+} \mathbf{r}_{-}^{k}=k \mathbf{r}_{+}^{k-1}$, but the second condition can not be written on this form because $\mathbf{P}_{-}$is singular.

Since the interior stencil is $2 p$ th order accurate and the weights in the scalar products equal one in the interior, we only need to enforce (3.8) and (3.9) for the first and last $m$ rows. Furthermore, symmetry considerations imply that the last $m$ rows of $\mathbf{Q}_{+}$and $\mathbf{Q}_{-}$can be constructed from the first $m$ rows of the same matrix. We therefore only need to consider the first $m$ rows of (3.8) and (3.9).

Because the first and last diagonal elements of $\mathbf{P}_{-}$are zero, the first and last columns of $\mathbf{Q}_{-}^{T}=\mathbf{D}_{-}^{T} \mathbf{P}_{-}$are zero. The SBP property (3.5) implies that the only non-zero element in the first column of $\mathbf{Q}_{+}=\mathbf{P}_{+} \mathbf{D}_{+}$is $\left(\mathbf{Q}_{+}\right)_{00}=-1$. By the same argument, the only non-zero element in its last column is $\left(\mathbf{Q}_{+}\right)_{N, N+1}=1$.

For example, the interior difference stencil for 4 th order interior accuracy $(p=2)$ correspond to

$$
\left.\mathbf{Q}_{+} \boldsymbol{\phi}_{+}\right|_{j}=\frac{1}{24} \phi_{j-3 / 2}-\frac{9}{8} \phi_{j-1 / 2}+\frac{9}{8} \phi_{j+1 / 2}-\frac{1}{24} \phi_{j+3 / 2} .
$$


The above considerations imply that $\mathbf{Q}_{+}$must have the following structure:

$$
\mathbf{Q}_{+}=\left[\begin{array}{cccccccccr}
-1 & q_{01} & q_{02} & q_{03} & q_{04} & 0 & 0 & 0 & 0 & 0 \\
0 & q_{11} & q_{12} & q_{13} & q_{14} & 0 & 0 & 0 & 0 & 0 \\
0 & q_{21} & q_{22} & q_{23} & q_{24} & 0 & 0 & 0 & 0 & 0 \\
0 & q_{31} & q_{32} & q_{33} & q_{34} & 1 / 24 & 0 & 0 & 0 & 0 \\
0 & 0 & 0 & 1 / 24 & -9 / 8 & 9 / 8 & -1 / 24 & 0 & 0 & 0 \\
0 & 0 & 0 & 0 & -1 / 24 & -q_{34} & -q_{33} & -q_{32} & -q_{31} & 0 \\
0 & 0 & 0 & 0 & 0 & -q_{24} & -q_{23} & -q_{22} & -q_{21} & 0 \\
0 & 0 & 0 & 0 & 0 & -q_{14} & -q_{13} & -q_{12} & -q_{11} & 0 \\
0 & 0 & 0 & 0 & 0 & -q_{04} & -q_{03} & -q_{02} & -q_{01} & 1
\end{array}\right]
$$

To conserve space, the matrix only has one row where the interior stencil is used. In practice, that stencil is used at all interior points, which gives the matrix a banded structure. For symmetry reasons, the $4 \times 4$ block of unknown coefficents $q_{i j}$ at the top of $\mathbf{Q}_{+}$also occurs at the bottom, but with opposite sign and reversed rows and columns. The structure of the matrices $\mathbf{Q}_{+}$and $\mathbf{Q}_{-}$ is discussed in detail in section 3.3.

The matrix (3.10) has 16 unknown coefficients $q_{i j}$. There are also 4 unknown weights in $\mathbf{P}_{+}$, and 4 unknown weights of $\mathbf{P}_{-}$. These 24 unknowns are determined by satisfying the first 4 equations of (3.8), the first 4 equations of (3.9), and the SBP constraint (3.5). This leads to an under-determined system where the solution is chosen to minimize the spectral radius of $\mathbf{D}_{+}$and $\mathbf{D}_{-}$, their truncation errors, or some combination thereof.

We have derived SBP operators with $4^{t h}, 6^{\text {th }}$, and $8^{\text {th }}$ order interior accuracy. MATLAB implementations of these operators are available at: http://github. com/ooreilly/sbp/.

\subsection{Enforcement of boundary conditions}

To demonstrate how to enforce boundary conditions, we consider the diffusion equation in one dimension as a motivating example. Here we focus on 
the diffusion operator in a Cartesian coordinate system. The key results from this section will be used to enforce the no-slip boundary condition. We contend with the polar coordinate singularity in the axisymmetric case in the subsequent section.

Consider the diffusion equation

$$
\frac{\partial u}{\partial t}=\frac{\partial^{2} u}{\partial x^{2}}, 0 \leq x \leq 1, t \geq 0,
$$

where $u=u(x, t)$. The energy method is applied by multiplying (3.11) with $u$ and integrating over the domain, and integrating by parts:

$$
\frac{1}{2} \frac{d\|u\|^{2}}{d t}=\left[u \frac{\partial u}{\partial x}\right]_{x=0}^{1}-\int_{x=0}^{1}\left(\frac{\partial u}{\partial x}\right)^{2} d x
$$

where $\|u\|^{2}=\int_{0}^{1} u^{2} d x$. We obtain an energy estimate for this problem by enforcing appropriate boundary conditions at each endpoint. We restrict attention to homogeneous Dirichlet conditions:

$$
u(0, t)=0 \quad \text { and } \quad u(1, t)=0,
$$

or homogeneous Neumann conditions:

$$
\frac{\partial u}{\partial x}(0, t)=0, \quad \text { and } \quad \frac{\partial u}{\partial x}(1, t)=0 .
$$

Either choice of these conditions results in the energy rate

$$
\frac{1}{2} \frac{d\|u\|^{2}}{d t}=-\int_{x=0}^{1}\left(\frac{\partial u}{\partial x}\right)^{2} d x \leq 0 .
$$

Next, we construct a semi-discrete approximation of (3.11) using the staggered SBP operators and enforce the boundary conditions by injection. To separate out the boundary points from the interior points we introduce the matrix $\mathbf{S}$,

$$
\mathbf{S}=\left[\begin{array}{cccccc}
0 & 1 & 0 & & & \\
& 0 & 1 & 0 & & \\
& & \ddots & \ddots & \ddots & \\
& & & 0 & 1 & 0
\end{array}\right] \in \mathbb{R}^{N \times(N+2)}
$$


Let $\mathbf{v}_{-}=\left[\begin{array}{llllll}v_{0} & v_{1 / 2} & v_{3 / 2} & \ldots & v_{N-1 / 2} & v_{N}\end{array}\right]^{T} \in \mathbb{R}^{(N+2)}$ denote a staggered grid function and let $\overline{\mathbf{v}}=\left[\begin{array}{llll}v_{1 / 2} & v_{3 / 2} & \ldots & v_{N-1 / 2}\end{array}\right]^{T} \in \mathbb{R}^{N}$ be the corresponding interior grid function. We have

$$
\overline{\mathbf{v}}=\mathbf{S v}_{-}, \quad \mathbf{v}_{-}=\left[\begin{array}{c}
v_{0} \\
\overline{\mathbf{v}} \\
v_{N}
\end{array}\right] .
$$

More generally, SA removes the first and last rows from $\mathbf{A} \in \mathbb{R}^{(N+2) \times n}$, and $\mathbf{B S}^{T}$ removes the first and last columns from $\mathbf{B} \in \mathbb{R}^{m \times(N+2)}$. Thus, the diagonal matrix

$$
\overline{\mathbf{P}}_{-}=\mathbf{S P}_{-} \mathbf{S}^{T}=\operatorname{diag}\left(\tilde{w}_{1 / 2}, \tilde{w}_{3 / 2}, \ldots, \tilde{w}_{N-1 / 2}\right) \in \mathbb{R}^{N \times N},
$$

is positive definite. Because of the accuracy constraint (3.9), the first and last rows of $\mathbf{Q}_{-}$are zero. We can therefore only define the difference approximation at the interior staggered points, i.e., $\overline{\mathbf{u}}=\overline{\mathbf{D}}_{-} \mathbf{v}_{+}$, where

$$
\overline{\mathbf{D}}_{-}=\overline{\mathbf{P}}_{-}^{-1} \overline{\mathbf{Q}}_{-} \in \mathbb{R}^{N \times(N+1)}, \quad \overline{\mathbf{Q}}_{-}=\mathbf{S Q}_{-} \in \mathbb{R}^{N \times(N+1)} .
$$

To separate out boundary and interior points when evaluating $\mathbf{D}_{+} \mathbf{v}_{-}$, we notice that $\mathbf{Q}_{+}$has the structure

$$
\mathbf{Q}_{+}=\left[\begin{array}{lll}
-\mathbf{e}_{0} & \overline{\mathbf{Q}}_{+} & \mathbf{e}_{N}
\end{array}\right], \quad \overline{\mathbf{Q}}_{+}=\mathbf{Q}_{+} \mathbf{S}^{T},
$$

where $\mathbf{e}_{0}=\left[\begin{array}{lllll}1 & 0 & 0 & \ldots & 0\end{array}\right]^{T} \in \mathbb{R}^{(N+1)}$ and $\mathbf{e}_{N}=\left[\begin{array}{lllll}0 & 0 & \ldots & 0 & 1\end{array}\right]^{T} \in \mathbb{R}^{(N+1)}$. Because $\mathbf{D}_{+}=\mathbf{P}_{+}^{-1} \mathbf{Q}_{+}$,

$$
\begin{aligned}
\mathbf{D}_{+} \mathbf{v}_{-}=\mathbf{P}_{+}^{-1}\left(-v_{0} \mathbf{e}_{0}+v_{N} \mathbf{e}_{N}+\overline{\mathbf{Q}}_{+} \overline{\mathbf{v}}\right) & \\
= & : \mathbf{P}_{+}^{-1}\left(-v_{0} \mathbf{e}_{0}+v_{N} \mathbf{e}_{N}\right)+\overline{\mathbf{D}_{+}} \overline{\mathbf{v}}, \quad \overline{\mathbf{D}}_{+}=\mathbf{P}_{+}^{-1} \overline{\mathbf{Q}}_{+} .
\end{aligned}
$$

Using the above definitions, the semi-discrete approximation of (3.11) becomes

$$
\frac{d \overline{\mathbf{v}}}{d t}=\overline{\mathbf{D}}_{-}\left[\mathbf{P}_{+}^{-1}\left(v_{N} \mathbf{e}_{N}-v_{0} \mathbf{e}_{0}\right)+\overline{\mathbf{D}}_{+} \overline{\mathbf{v}}\right],
$$

where $v_{N}$ and $v_{0}$ are determined from the boundary conditions.

The semi-discrete approximation is stable because it satifies an energy rate equation analogous to (3.15), as is made precise by the following lemma. 
Lemma 3.1. The semi-discrete approximation (3.20) satisfies the energy rate equation

$$
\frac{1}{2} \frac{d\|\overline{\mathbf{v}}\|_{-}^{2}}{d t}=-\left(\overline{\mathbf{D}_{+}} \overline{\mathbf{v}}\right)_{N} v_{N}+\left(\overline{\mathbf{D}_{+}} \overline{\mathbf{v}}\right)_{0} v_{0}-\left(\overline{\mathbf{D}}_{+} \overline{\mathbf{v}}\right)^{T} \mathbf{P}_{+}\left(\overline{\mathbf{D}}_{+} \overline{\mathbf{v}}\right) .
$$

Proof. Our proof is based on the energy method. Taking the time derivative of $\|\overline{\mathbf{v}}\|_{-}^{2}$, using (3.20), and the above definition of $\overline{\mathbf{D}}_{-}$, results in

$$
\frac{1}{2} \frac{d\|\overline{\mathbf{v}}\|_{-}^{2}}{d t}=\overline{\mathbf{v}}^{T} \overline{\mathbf{Q}}_{-} \mathbf{P}_{+}^{-1}\left(v_{N} \mathbf{e}_{N}-v_{0} \mathbf{e}_{0}\right)+\overline{\mathbf{v}}^{T} \overline{\mathbf{Q}}_{-} \overline{\mathbf{D}}_{+} \overline{\mathbf{v}} .
$$

Because of (3.18), it follows from the SBP property (3.5) that

$$
\overline{\mathbf{Q}}_{+}+\overline{\mathbf{Q}}_{-}^{T}=0 .
$$

Hence, the definition of $\overline{\mathbf{D}}_{+}$gives $\overline{\mathbf{v}}^{T} \overline{\mathbf{Q}}_{-} \mathbf{P}_{+}^{-1}=-\left(\overline{\mathbf{D}}_{+} \overline{\mathbf{v}}\right)^{T}$ and we arrive at (3.21).

Corollary 3.2. (Dirichlet boundary conditions) If the initial conditions satisfy $v_{0}=0$ and $v_{N}=0$, the semi-discrete approximation

$$
\frac{d \overline{\mathbf{v}}}{d t}=\overline{\mathbf{D}}_{-} \overline{\mathbf{D}}_{+} \overline{\mathbf{v}}, \quad t \geq 0,
$$

satisfies homogeneous Dirichlet boundary conditions for all $t>0$, and is stable.

Proof. The homogeneous Dirichlet condition (3.13) are discretized by

$$
v_{0}=0 \quad \text { and } \quad v_{N}=0 .
$$

Inserting (3.25) into (3.20), leads to (3.24). Because the scheme does not change the boundary values of $\mathbf{v}_{-}$, the Dirichlet conditions are satisfied for all $t>0$. By inserting (3.25) into (3.21), it follows that this semi-discrete approximation satisfies the energy rate equation

$$
\frac{1}{2} \frac{d\|\overline{\mathbf{v}}\|_{-}^{2}}{d t}=-\left(\overline{\mathbf{D}_{+}} \overline{\mathbf{v}}\right)^{T} \mathbf{P}_{+}\left(\overline{\mathbf{D}}_{+} \overline{\mathbf{v}}\right) \leq 0,
$$

which proves energy stability. 
Corollary 3.3. (Neumann boundary conditions) The semi-discrete approximation

$$
\frac{d \overline{\mathbf{v}}}{d t}=\overline{\mathbf{D}}_{-}\left[-\left(\overline{\mathbf{D}}_{+} \overline{\mathbf{v}}\right)_{N} \mathbf{e}_{N}-\left(\overline{\mathbf{D}_{+}} \overline{\mathbf{v}}\right)_{0} \mathbf{e}_{0}+\overline{\mathbf{D}}_{+} \overline{\mathbf{v}}\right],
$$

is stable and enforces the discrete homogeneous Neumann conditions

$$
\left(\mathbf{D}_{+} \mathbf{v}_{-}\right)_{0}=0 \text { and }\left(\mathbf{D}_{+} \mathbf{v}_{-}\right)_{N}=0 \text {. }
$$

Proof. The discrete boundary conditions are equivalent to $\left(\mathbf{Q}_{+} \mathbf{v}_{-}\right)_{0}=0$ and $\left(\mathbf{Q}_{+} \mathbf{v}_{-}\right)_{N}=0$. Because the matrix $\mathbf{Q}_{+}$has the decomposition (3.18), these conditions are satisfied by taking

$$
-v_{0}+\left(\overline{\mathbf{Q}}_{+} \overline{\mathbf{v}}\right)_{0}=0 \quad \text { and } \quad v_{N}+\left(\overline{\mathbf{Q}}_{+} \overline{\mathbf{v}}\right)_{N}=0 .
$$

Inserting (3.27) into (3.20), leads to (3.26). By inserting (3.27) into (3.21), it follows that this semi-discrete approximation satisfies the energy rate

$$
\begin{aligned}
& \frac{1}{2} \frac{d\|\overline{\mathbf{v}}\|_{-}^{2}}{d t}=\left(\overline{\mathbf{D}}_{+} \overline{\mathbf{v}}\right)_{N}^{2}\left(\mathbf{P}_{+}\right)_{N N}+\left(\overline{\mathbf{D}_{+}} \overline{\mathbf{v}}\right)_{0}^{2}\left(\mathbf{P}_{+}\right)_{00}-\left(\overline{\mathbf{D}_{+}} \overline{\mathbf{v}}\right)^{T} \mathbf{P}_{+}\left(\overline{\mathbf{D}}_{+} \overline{\mathbf{v}}\right) \\
& =-\sum_{j=1}^{N-1}\left(\overline{\mathbf{D}}_{+} \overline{\mathbf{v}}\right)_{j}^{2}\left(\mathbf{P}_{+}\right)_{j j} \leq 0 .
\end{aligned}
$$

Thus the discretization is energy stable.

\subsection{Construction of the radial diffusion operator}

We now return to the model problem in cylindrical coordinates. By focusing only on the radial and time dependence of $v$, the diffusive part of the momentum balance expression (2.3) can be expressed in the form

$$
\frac{\partial v}{\partial t}=\frac{\epsilon}{r} \frac{\partial}{\partial r}\left(r \frac{\partial v}{\partial r}\right)
$$

for $v=v(t, r)$ and with the no-slip boundary condition

$$
v(t, r=1)=0 .
$$

We approximate (3.28) by

$$
\frac{d \overline{\mathbf{v}}}{d t}=\epsilon \mathbf{D}_{2} \mathbf{v}
$$


The difference operator $\mathbf{D}_{2}$ approximates the radial component of the Laplacian and is constructed along the lines of (3.20):

$$
\mathbf{D}_{2} \mathbf{v}_{-}=\overline{\mathbf{R}}_{-}^{-1} \overline{\mathbf{D}}_{-}\left[\mathbf{P}_{+}^{-1}\left(r_{N} v_{N} \mathbf{e}_{N}-r_{0} v_{0} \mathbf{e}_{0}\right)+\mathbf{R}_{+} \overline{\mathbf{D}}_{+} \overline{\mathbf{v}}\right] \in \mathbb{R}^{N},
$$

where $\mathbf{R}_{+}=\operatorname{diag}\left(\mathbf{r}_{+}\right) \in \mathbb{R}^{(N+1) \times(N+1)}$ and $\overline{\mathbf{R}}_{-}=\operatorname{diag}\left(\overline{\mathbf{r}}_{-}\right) \in \mathbb{R}^{N \times N}, \overline{\mathbf{r}}_{-}=$ $S \mathbf{r}_{-}$. This difference approximation avoids the computation of the numerical solution at the coordinate singularity $r=0$ and also injects the no-slip boundary condition (3.29) by taking $v_{N}=0$. Since $r_{0}=0$, it is not possible to specify $v_{0}$. After injecting the no-slip boundary condition, we get

$$
\mathbf{D}_{2} \equiv \overline{\mathbf{R}}_{-}^{-1} \overline{\mathbf{D}}_{-} \mathbf{R}_{+} \overline{\mathbf{D}}_{+} \in \mathbb{R}^{N \times N}
$$

and

$$
\frac{d \overline{\mathbf{v}}}{d t}=\epsilon \mathbf{D}_{2} \overline{\mathbf{v}}
$$

Hence, the dependent variables consist of the interior points in the staggered grid.

In the second order case $(p=1)$, the radial diffusion operator becomes

$$
\mathbf{D}_{2}=\frac{1}{\Delta r^{2}}\left[\begin{array}{ccccc}
-\frac{r_{1}}{r_{1 / 2}} & \frac{r_{1}}{r_{1 / 2}} & & & \\
\frac{r_{1}}{r_{3 / 2}} & -\frac{r_{1}+r_{2}}{r_{3 / 2}} & \frac{r_{2}}{r_{3 / 2}} & & \\
& \ddots & \ddots & \ddots & \\
& & \frac{r_{N-2}}{r_{N-3 / 2}} & -\frac{r_{N-2}+r_{N-1}}{r_{N-3 / 2}} & \frac{r_{N-1}}{r_{N-3 / 2}} \\
& & & \frac{r_{N-1}}{r_{N-1 / 2}} & -\frac{r_{N-1+2 r_{N}}}{r_{N-1 / 2}}
\end{array}\right] .
$$

\section{Semi-discrete approximation of the model problem}

We now implement the discrete operator (3.30) in the context of the model problem defined in section 2. This $2-\mathrm{D}$ problem requires the construction of a grid in the $z$ direction with corresponding $\partial / \partial z$ operators in addition to the radial grids and associated difference operators defined in section 3. We define circulant first derivative operators that act in the $z$ direction and reflect the periodicity condition at the bottom $(z=0)$ and top $(z=2 \pi)$ of the domain. 
By discretizing the 2-D problem using SBP operators, we prove energy stability for the system.

\subsection{Grid definitions}

To create a discretization of our 2-D model problem, we use the $\mathbf{r}_{+}$grid defined in section 3 , as well as the truncated grid $\overline{\mathbf{r}}_{-}=\mathbf{S r}_{-}$. The number of grid points on $\overline{\mathbf{r}}_{-}$is $N_{r}$. The $z$ direction can be discretized using either a single grid for both $p$ and $v$ or using staggered grids. Since the dispersion error is smaller for central first derivatives on staggered grids compared to collocated grids, we discretize the $z$ direction using staggered grids as well. The grids are given by:

$$
\mathbf{z}_{+}=\left[z_{0} z_{1} z_{2} \ldots z_{N_{z}-1}\right]^{T}, \quad \mathbf{z}_{-}=\left[z_{\frac{1}{2}} z_{\frac{3}{2}} \ldots z_{N_{z}-\frac{1}{2}}\right]^{T}
$$

where

$$
z_{j}=j \Delta z \text { for } 0 \leq j \leq N_{z}-1, z_{j+1 / 2}=\left(j+\frac{1}{2}\right) \Delta z \text { for } 0 \leq j \leq N_{z}-1
$$

for step size $\Delta z=2 \pi / N_{z}$, where $N_{z}$ is the number of grid points in the $z$ direction. It is important to note that these grids do not have the same structure as the staggered grids in the radial direction (i.e., the + and - grids do not share the same endpoints). The point $z_{N_{z}}$ is not stored, since by the periodicity condition it is equal to $z_{0}$.

\subsection{Numerical scheme}

The velocity $\overline{\mathbf{v}}$ (along with other 2 -D fields) is stored in a vector using column-major ordering (along the radial direction first):

$$
\overline{\mathbf{v}}=\left[\vec{v}_{\frac{1}{2}} \vec{v}_{\frac{3}{2}} \ldots \vec{v}_{N_{z}-\frac{1}{2}}\right]^{T} \in \mathbb{R}^{\left(N_{r} N_{z}\right)} .
$$

The vectors $\vec{v}_{j}$ containing the velocity values along the radial direction at some $z_{j}$ are

$$
\vec{v}_{j}=\left[v_{\frac{1}{2}, j} v_{\frac{3}{2}, j} \ldots v_{N_{r}-\frac{1}{2}, j}\right] \in \mathbb{R}^{1 \times N_{r}}
$$


where $v_{i j}=v\left(t, z_{j}, r_{i}\right)$. The cross-sectionally averaged velocity is stored on the $\mathbf{z}_{-}$grid and the pressure on the $\mathbf{z}_{+}$grid:

$$
\begin{aligned}
& \mathbf{p}=\left[p_{0} p_{1} p_{2} \ldots p_{N_{z}-1}\right]^{T} \in \mathbb{R}^{N_{z}}, \\
& \mathbf{u}=\left[\begin{array}{ll}
u_{1 / 2} & u_{3 / 2} \ldots u_{N_{z}-1 / 2}
\end{array}\right]^{T} \in \mathbb{R}^{N_{z}},
\end{aligned}
$$

where $p_{j}=p\left(t, z_{j}\right)$ and $u_{j}=u\left(t, z_{j}\right)$; neither depends on $r$. The grid points at which the fields are stored are shown in Figure 2.

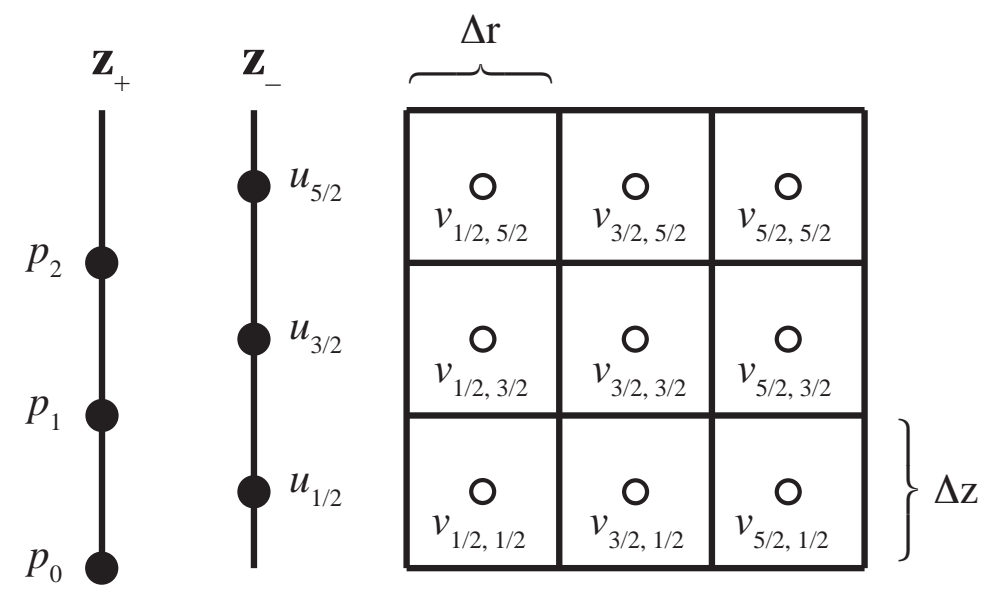

Figure 2: The mesh used in the implementation of the model problem. $\mathbf{p}$ and $\mathbf{u}$ are $1 \mathrm{D}$ fields stored on the $\mathbf{z}_{+}$and $\mathbf{z}_{-}$grids respectively, while $\mathbf{v}$ is a $2-\mathrm{D}$ field stored on the $\overline{\mathbf{r}}_{-}$and $\mathbf{z}_{-}$ grids.

In the $z$ direction, the derivatives of the continuous functions $u(t, z)$ and $p(t, z)$ are approximated using standard central difference operators $\mathbf{D}_{z^{-}}$and $\mathbf{D}_{z^{+}}$on a staggered, periodic grid. The operator $\mathbf{D}_{z^{-}}$acts on quantities stored on the $\mathbf{z}_{+}$grid, with the resulting product stored on the $\mathbf{z}_{-}$grid, while $\mathbf{D}_{z^{+}}$ acts on quantities stored on the $\mathbf{z}_{-}$grid, with the product stored on the $\mathbf{z}_{+}$ grid. Due to periodicity in the $z$ direction, the difference operators $\mathbf{D}_{z^{-}}$and $\mathbf{D}_{z^{+}}$satisfy the SBP property:

$$
\mathbf{D}_{z^{+}}=-\mathbf{D}_{z^{-}}^{T}
$$

Using these operators, the $z$ derivative of the continuous function $p(t, z)$ is ap- 
proximated in terms of the discrete function $\mathbf{p}$ (stored on the $\mathbf{z}_{+}$grid) as

$$
\frac{\partial p}{\partial z} \approx \mathbf{D}_{z}-\mathbf{p}
$$

To apply these one-dimensional difference operators to a two-dimensional field (such as $\mathbf{v}$ ), we must apply them at each point in the radial direction. This is accomplished by using the Kronecker product, defined by

$$
\mathbf{A} \otimes \mathbf{B}=\left[\begin{array}{ccc}
a_{11} \mathbf{B} & \ldots & a_{1 n} \mathbf{B} \\
\vdots & \ddots & \vdots \\
a_{m 1} \mathbf{B} & \ldots & a_{m n} \mathbf{B}
\end{array}\right]
$$

We can then apply the operator $\mathbf{D}_{2}$, which acts in the radial direction only, to the two dimensional velocity field $\mathbf{v}$ to approximate the viscous term in the governing equations (2.3):

$$
\frac{1}{r} \frac{\partial}{\partial r}\left(r \frac{\partial v(t, z, r)}{\partial r}\right) \approx\left(\mathbf{I}_{z} \otimes \mathbf{D}_{2}\right) \overline{\mathbf{v}}
$$

where $\mathbf{I}_{z} \in \mathbb{R}^{N_{z} \times N_{z}}$ is an identity matrix.

The semi-discrete form of the governing equations (2.1) and (2.3) can thus be written as

$$
\begin{aligned}
& \frac{d \mathbf{p}}{d t}=-\mathbf{D}_{z+} \mathbf{u} \\
& \frac{d \overline{\mathbf{v}}}{d t}=-\left(\mathbf{D}_{z^{-}} \mathbf{p} \otimes \mathbf{e}_{-}\right)+\epsilon\left(\mathbf{I}_{z} \otimes \mathbf{D}_{2}\right) \overline{\mathbf{v}},
\end{aligned}
$$

where $\mathbf{e}_{-}=\left[\begin{array}{llll}1 & 1 & \ldots & 1\end{array}\right]^{T} \in \mathbb{R}^{N_{r}}$. As in the continuous case, we have defined the cross-sectionally averaged velocity

$$
\mathbf{u}=\left(\mathbf{I}_{z} \otimes \mathbf{w}^{T}\right) \overline{\mathbf{v}} \approx 2 \int_{0}^{1} v(t, z, r) r d r
$$

where $\mathbf{w}$ is the cross-sectional averaging operator, constructed using the quadrature rule $\mathbf{P}_{-}$on the staggered radial grid:

$$
\mathbf{w}^{T}=2 \mathbf{e}_{-}^{T} \overline{\mathbf{R}}_{-} \overline{\mathbf{P}}_{-} \in \mathbb{R}^{1 \times N_{r}} .
$$

The discrete cross-sectionally averaged velocity $\mathbf{u}(4.8)$ approximates the continuous cross-sectionally averaged velocity $u(t, z)$ defined in $(2.2)$. 


\subsection{Energy balance}

To compute the discrete energy, we introduce a two-dimensional discrete grid function $\mathbf{a}_{-}$defined on the $\overline{\mathbf{r}}_{-}$and $\mathbf{z}_{-}$grids in the same manner as $\mathbf{v}$ in (4.2) and (4.3), as well as $\mathbf{a}_{+}$defined on the $\mathbf{r}_{+}$and $\mathbf{z}_{+}$grids. We then define discrete norms for two-dimensional fields on both radial grids:

$$
\left\|\mathbf{a}_{-}\right\|_{\Omega^{-}}^{2}=\mathbf{a}_{-}^{T} \mathbf{P}_{\Omega^{-}} \mathbf{a}_{-}, \quad\left\|\mathbf{a}_{+}\right\|_{\Omega^{+}}^{2}=\mathbf{a}_{+}^{T} \mathbf{P}_{\Omega^{+}} \mathbf{a}_{+},
$$

where

$$
\mathbf{P}_{\Omega^{-}}=\mathbf{P}_{z} \otimes \overline{\mathbf{R}}_{-} \overline{\mathbf{P}}_{-} \quad \text { and } \quad \mathbf{P}_{\Omega^{+}}=\mathbf{P}_{z} \otimes \mathbf{R}_{+} \mathbf{P}_{+}
$$

are quadrature rules approximating the area of $\Omega$. Since the solution is periodic

in the $z$ direction, $\mathbf{P}_{z}=\Delta z \mathbf{I}_{z} \in \mathbb{R}^{N_{z} \times N_{z}}$. This quadrature rule is therefore the same for both grids $\mathbf{z}_{-}$and $\mathbf{z}_{+}$.

Using these norms, the discrete energy is defined as

$$
E_{h}=\frac{1}{2}\|\overline{\mathbf{v}}\|_{\Omega^{-}}^{2}+\frac{1}{2}\left\|\mathbf{p} \otimes \mathbf{e}_{-}\right\|_{\Omega^{-}}^{2}=\frac{1}{2} \overline{\mathbf{v}}^{T}\left(\mathbf{P}_{z} \otimes \overline{\mathbf{R}}_{-} \overline{\mathbf{P}}_{-}\right) \overline{\mathbf{v}}+\frac{1}{4} \mathbf{p}^{T} \mathbf{P}_{z} \mathbf{p} .
$$

The result $\left\|\mathbf{p} \otimes \mathbf{e}_{-}\right\|_{\Omega^{-}}^{2}=\mathbf{p}^{T} \mathbf{P}_{z} \mathbf{p} / 2$ follows from the definition of the crosssectionally averaged operator (4.9) :

$$
\begin{aligned}
\left\|\mathbf{p} \otimes \mathbf{e}_{-}\right\|_{\Omega_{-}}^{2} & =\left(\mathbf{p}^{T} \otimes \mathbf{e}_{-}^{T}\right)\left(P_{z} \otimes \overline{\mathbf{R}}_{-} \overline{\mathbf{P}}_{-}\right)\left(\mathbf{p} \otimes \mathbf{e}_{-}\right) \\
& =\left(\mathbf{p}^{T} P_{z} \mathbf{p} \otimes \mathbf{e}_{-}^{T} \overline{\mathbf{R}}_{-} \overline{\mathbf{P}}_{-} \mathbf{e}_{-}\right) \\
& =\frac{1}{2}\left(\mathbf{p}^{T} P_{z} \mathbf{p} \otimes \mathbf{w}^{T} \mathbf{e}_{-}\right) \\
& =\frac{1}{2} \mathbf{p}^{T} P_{z} \mathbf{p}
\end{aligned}
$$

where we used the fact that

$$
\mathbf{w}^{T} \mathbf{e}_{-}=2 \int_{r=0}^{r=1} r d r=1
$$

(see Appendix A).

Using the fact that the quadrature rules are symmetric, positive definite matrices, we compute the energy rate for the system by taking the time derivative 
of (4.11) and inserting (4.6) and (4.7) into the result, which yields

$$
\begin{aligned}
\frac{d E_{h}}{d t}= & -\frac{1}{2} \mathbf{p}^{T} \mathbf{P}_{z} \mathbf{D}_{z+} \mathbf{u}-\overline{\mathbf{v}}^{T}\left(\mathbf{P}_{z} \mathbf{D}_{z-} \mathbf{p} \otimes \overline{\mathbf{R}}_{-} \overline{\mathbf{P}}_{-} \mathbf{e}_{-}\right) \\
& +\epsilon \overline{\mathbf{v}}^{T}\left(\mathbf{P}_{z} \otimes \overline{\mathbf{P}}_{-} \overline{\mathbf{D}}_{-} \mathbf{R}_{+} \overline{\mathbf{D}}_{+}\right) \overline{\mathbf{v}} .
\end{aligned}
$$

From the definition of the cross-sectionally averaged operator (4.9), we obtain

$$
\begin{aligned}
\overline{\mathbf{v}}^{T}\left(\mathbf{P}_{z} \mathbf{D}_{z-} \mathbf{p} \otimes \overline{\mathbf{R}}_{-} \overline{\mathbf{P}}_{-} \mathbf{e}_{-}\right) & =\mathbf{p}^{T} \mathbf{D}_{z^{-}}^{T} \mathbf{P}_{z}\left(\mathbf{I}_{z} \otimes \mathbf{e}_{-}^{T} \overline{\mathbf{R}}_{-} \overline{\mathbf{P}}_{-}\right) \overline{\mathbf{v}} \\
& =\frac{1}{2} \mathbf{p}^{T} \mathbf{D}_{z^{-}}^{T} \mathbf{P}_{z}\left(\mathbf{I}_{z} \otimes \mathbf{w}^{T}\right) \overline{\mathbf{v}} \\
& =\frac{1}{2} \mathbf{p}^{T} \mathbf{D}_{z^{-}}^{T} \mathbf{P}_{z} \mathbf{u} .
\end{aligned}
$$

Then (4.12) becomes

$$
\frac{d E_{h}}{d t}=-\frac{1}{2} \mathbf{p}^{T}\left(\mathbf{P}_{z} \mathbf{D}_{z^{+}}+\mathbf{D}_{z^{-}}^{T} \mathbf{P}_{z}\right) \mathbf{u}+\epsilon \overline{\mathbf{v}}^{T}\left(\mathbf{P}_{z} \otimes \overline{\mathbf{P}}_{-} \overline{\mathbf{D}}_{-} \mathbf{R}_{+} \overline{\mathbf{D}}_{+}\right) \overline{\mathbf{v}}
$$

Due to the SBP property of the $z$ operators given in (4.4), the first two terms on the right-hand side of (4.13) cancel each other out. Applying the SBP property for the radial operators (3.5), we substitute for $\overline{\mathbf{D}}_{-}$to simplify (4.13):

$$
\frac{d E_{h}}{d t}=-\epsilon \overline{\mathbf{v}}^{T}\left(\mathbf{P}_{z} \otimes \overline{\mathbf{D}}_{+}^{T} \mathbf{P}_{+} \mathbf{R}_{+} \overline{\mathbf{D}}_{+}\right) \overline{\mathbf{v}}+\epsilon \overline{\mathbf{v}}^{T}\left(\mathbf{P}_{z} \otimes \overline{\mathbf{B}}^{T} \mathbf{R}_{+} \overline{\mathbf{D}}_{+}\right) \overline{\mathbf{v}}
$$

The truncated boundary matrix $\overline{\mathbf{B}}^{T}$ is a zero matrix, so only the viscous dissipation term is left:

$$
\frac{d E_{h}}{d t}=-\epsilon\left\|\left(\mathbf{I}_{z} \otimes \overline{\mathbf{D}}_{+}\right) \overline{\mathbf{v}}\right\|_{\Omega^{+}}^{2}
$$

The scheme is stable if the right-hand side is non-positive. Stability is addressed in the following proposition:

Proposition 4.1. The discrete energy rate in (4.14) satisfies

$$
\frac{d E_{h}}{d t}=-\epsilon\left\|\left(\mathbf{I}_{z} \otimes \overline{\mathbf{D}}_{+}\right) \overline{\mathbf{v}}\right\|_{\Omega^{+}}^{2}<0,
$$

for $\epsilon>0$ and for all $\overline{\mathbf{v}} \neq 0$.

Proof. The proof is given Appendix B for the second order accurate difference approximation. The proposition is numerically verified to hold for higher orders as well. 


\section{Convergence tests}

We investigate the accuracy of our numerical scheme by constructing a smooth analytic solution with a harmonic pressure of the form

$$
p(t, z)=\operatorname{Re}\left(e^{i(z-\omega t)}\right)
$$

for complex $\omega$ to be determined below; $\operatorname{Re}(\omega)$ is the angular frequency of oscillation. We chose the nondimensional domain length in the axial direction to be exactly one wavelength, so the wavenumber $k$ is equal to 1 . Following [1], we solve the momentum balance to find the velocity $v(t, z, r)$ satisfying the no-slip boundary condition $v(t, z, r=1)=0$ as

$$
v(t, z, r)=\operatorname{Re}\left(\frac{1}{\omega}\left[1-\frac{J_{0}(\alpha r)}{J_{0}(\alpha)}\right] e^{i(z-\omega t)}\right),
$$

where $\alpha=-\sqrt{i \omega / \epsilon}$, and $J_{0}(x)$ is a Bessel function of the first kind. Figure 3a shows radial profiles of the velocity for $\epsilon=10^{-2}$ at a fixed depth $z=\pi$ after 0,1 , and 2 periods $T=2 \pi / \operatorname{Re}(\omega)$. The velocity retains the same profile but decreases in amplitude with each cycle due to viscous dissipation. In Figure $3 \mathrm{~b}$, profiles at $t=0$ are shown for different values of $\epsilon$. As $\epsilon$ is increased, the width of the viscous boundary layer increases. In the limit of large $\epsilon$ the flow is fully developed (the boundary layers meet at the center of the conduit to form a parabolic velocity profile), while as $\epsilon$ approaches 0 the flow approaches plug flow behavior.

Applying the width averaging (2.2) to the velocity given in (5.2), we obtain the cross-sectionally averaged velocity:

$$
u(t, z)=\operatorname{Re}\left(\frac{1}{\omega}\left[1-\frac{2}{\alpha} \frac{J_{1}(\alpha)}{J_{0}(\alpha)}\right] e^{i(z-\omega t)}\right),
$$

Upon satisfying the mass balance equation, we obtain the dispersion relation

$$
\omega^{2}=1-\frac{2}{\alpha} \frac{J_{1}(\alpha)}{J_{0}(\alpha)}
$$

In general, solving the dispersion relation for $\omega$ yields complex values, whose real and imaginary parts correspond to the oscillatory and decaying behavior 
of the wave, respectively. In the inviscid limit $(\epsilon=0), \omega \rightarrow \pm 1$, and waves propagate without energy loss. As $\epsilon$ is increased, the solutions to (5.4) acquire negative imaginary components, which correspond to dissipative behavior in the boundary layer. For large enough $\epsilon$, the real components of the solutions approach zero, producing overdamped wave behavior in which the waves decay without oscillating.
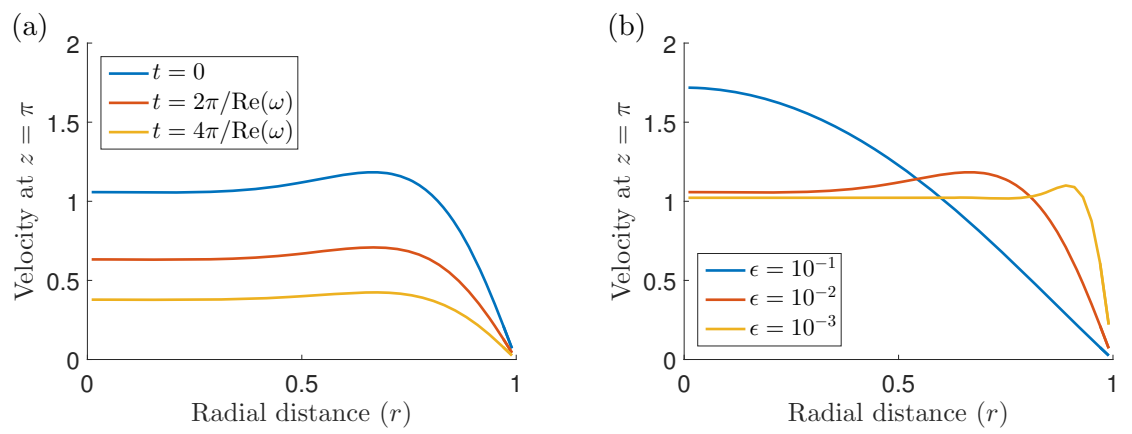

Figure 3: Radial profiles of the velocity $v$ at the middle of the conduit $(z=\pi)$. Figure (a) shows velocity profiles for $\epsilon=10^{-2}$ after 0,1 , and 2 periods, showing amplitude decay due to viscous dissipation. Figure (b) shows velocity profiles (at $t=0$ ) for different values of $\epsilon$. As $\epsilon$ is increased, the viscous boundary layer becomes wider.

\subsection{Optimal discretization}

Given a desired error level, or tolerance, and value of $\epsilon$, we determine the optimal discretization by comparing the error contributions due to discretization in the $r$ and $z$ directions. The norm of the error is computed using

$$
\|\mathbf{e}\|_{\Omega^{-}}^{2}=\left\|\mathbf{v}-\mathbf{v}^{*}\right\|_{\Omega^{-}}^{2}+\left\|\left(\mathbf{p}-\mathbf{p}^{*}\right) \otimes \mathbf{e}_{-}\right\|_{\Omega^{-}}^{2},
$$

where $\mathbf{v}^{*}$ and $\mathbf{p}^{*}$ is the velocity and pressure given by the analytic solution. The numerical solution is integrated in time using a low-storage fourth-order RungeKutta method [18] and the norm of the error is measured after one oscillation period $\left(t_{\max }=2 \pi / \operatorname{Re}(\omega)\right)$.

In Figure 4, the error contributions from the grid refinement in the $z$ and $r$ directions are visualized by fixing the grid refinement level in one direction and varying it in the other direction. In Figure $4 \mathrm{a}$, the value of $N_{z}$ is held 
fixed while $N_{r}$ is varied, while the reverse is true in Figure $4 \mathrm{~b}$. The optimal discretization for a desired error level is where the error contributions from the $r$ and $z$ discretizations are approximately equal, corresponding to the location where the error curves begin to plateau in the grid refinement plots.
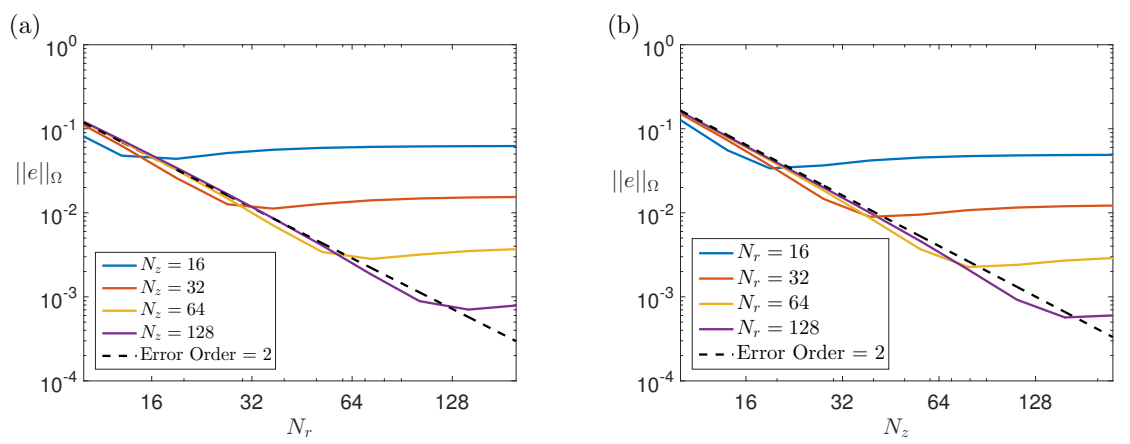

Figure 4: Error contributions from $r$ (a) and $z$ (b) grid refinement using 2nd order interior operators, with $\epsilon=10^{-2}$.

Decreasing $\epsilon$ narrows the boundary layer in which viscous effects act. To resolve this narrower boundary layer to a desired accuracy, the grid resolution in the radial direction must be increased. A grid refinement for $\epsilon=10^{-3}$ is shown in Figure 5b. Comparing Figures 5a and 5b, we see that when $\epsilon$ is reduced by a factor of 10 , the number of radial grid points $N_{r}$ needed for an optimal discretization for a given $N_{z}$ increases. Since the grid is uniform, the numerical solution in the center of the conduit becomes increasingly over resolved as the width of the boundary layer decreases. To avoid over resolving the numerical solution, one can adopt a coordinate transform in the radial direction that clusters most of the grid points in the boundary layer. 

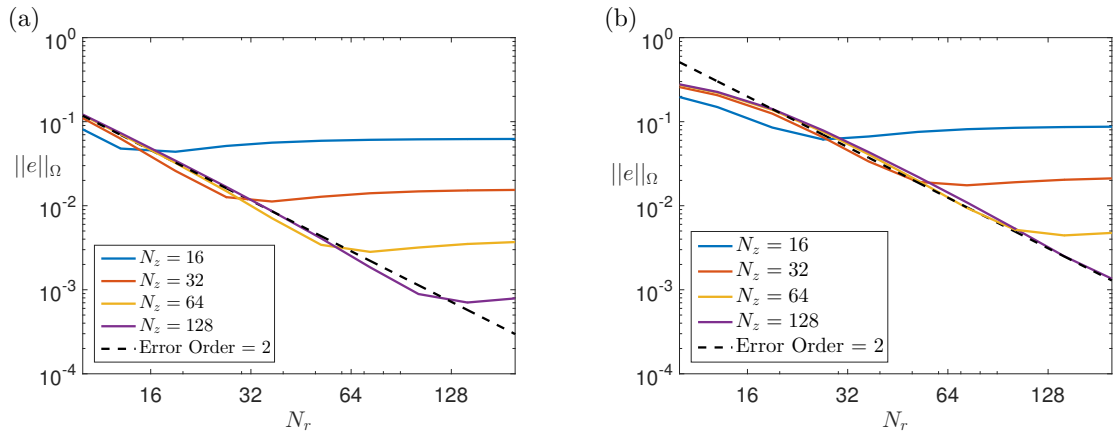

Figure 5: Comparison of error contributions from $z$ grid refinement using 2nd order interior operators with $\epsilon=10^{-2}$ (a) and $\epsilon=10^{-3}(\mathrm{~b})$. When $\epsilon$ is reduced, viscous effects are confined to a narrower boundary layer, so a higher resolution (larger $N_{r}$ ) is needed to achieve the same accuracy.

\subsection{Convergence rate}

We compute the accuracy of the simulation for operators of 2nd, 4th, 6th, and 8th order interior accuracy, with the results shown in Figure 6. The order of accuracy of the diffusion difference operator is $p$ at the boundary and $2 p$ in the interior. The expected global convergence rate is $p+1[19,20]$. By using factor of two refinements, we estimate the convergence rate using

$$
\text { Rate }=\log _{2}\left(\frac{\left\|\mathbf{e}_{j-1}\right\|_{\Omega^{-}}}{\left\|\mathbf{e}_{j}\right\|_{\Omega^{-}}}\right),
$$

where $\left\|\mathbf{e}_{j}\right\|_{\Omega^{-}}$denotes the error on a mesh $j$. For our convergence tests, we choose each successive mesh $j$ to have twice the number of grid points in both the radial and axial directions as the previous mesh $j-1$. Following the optimal discretization for $\epsilon=10^{-2}$ shown in Figure 4, we choose the same number of grid points in the radial and axial directions. The errors and convergence rates at each grid refinement are shown in table 1 . The error converges towards zero at the expected convergence rate $(p+1)$. 


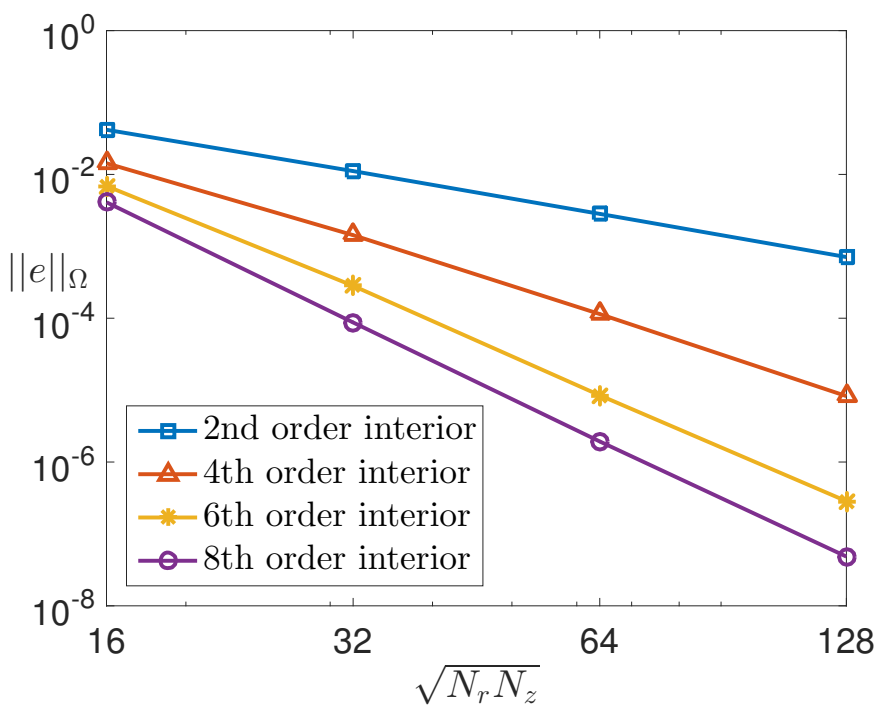

Figure 6: Convergence rates for 2nd through 8th order interior operators, with $\epsilon=10^{-2}$ and equal number of grid points in the $z$ and $r$ directions.

Table 1: Error magnitudes and rates for simulations using operators with 2nd through 8th order interior accuracy.

\begin{tabular}{|c|cc|cc|}
\hline$\sqrt{N_{z} N_{r}}$ & \multicolumn{2}{|c|}{ 2nd order interior accuracy } & \multicolumn{2}{c|}{4 th order interior accuracy } \\
& error magnitude & error rate & error magnitude & error rate \\
\hline 16 & $4.18 \times 10^{-2}$ & & $1.43 \times 10^{-2}$ & \\
32 & $1.11 \times 10^{-2}$ & 1.91 & $1.40 \times 10^{-3}$ & 3.32 \\
64 & $2.80 \times 10^{-3}$ & 1.98 & $1.14 \times 10^{-4}$ & 3.65 \\
128 & $7.09 \times 10^{-4}$ & 1.99 & $8.26 \times 10^{-6}$ & 3.79 \\
\hline \hline \multirow{2}{*}{$\sqrt{N_{z} N_{r}}$} & 6 th order interior accuracy & 8 th order interior accuracy \\
& error magnitude & error rate & error magnitude & error rate \\
\hline 16 & $6.90 \times 10^{-3}$ & & $4.10 \times 10^{-3}$ & \\
32 & $2.82 \times 10^{-4}$ & 4.61 & $8.73 \times 10^{-5}$ & 5.55 \\
64 & $8.47 \times 10^{-6}$ & 5.06 & $1.93 \times 10^{-6}$ & 5.50 \\
128 & $2.85 \times 10^{-7}$ & 4.89 & $4.84 \times 10^{-8}$ & 5.32 \\
\hline
\end{tabular}




\section{Application to acoustic-gravity waves in magmatic conduits}

The problem of axisymmetric wave propagation with viscous dissipation in a conduit arises in a range of different contexts, including arterial flow [1], aeroacoustic applications including airflow in human phonation [21], and acousticgravity waves in magmatic conduits [3]. Many of these application problems cannot be solved directly due to spatially variable material properties, timevarying boundary conditions, or multiphase fluids in the conduit.

The problem of wave propagation in magmatic conduits is of particular interest to us, and we introduce it here as a more complex problem on which we can apply the results developed in the previous sections. In this problem, $z$ is depth (positive up), and the magma properties are functions of depth. In Karlstrom and Dunham [3], a model is presented for acoustic-gravity waves in a cylindrical conduit filled with viscous, compressible magma in the presence of a gravitational field. Magma is a mixture of liquid melt and gas bubbles or volatiles that can be fully or partially dissolved in the melt according to pressure-dependent solubility laws. The model is motivated by observations at Kileaua Volcano, Hawaii, where an open lava lake sits atop a magma column extending down at least a few kilometers. Huge rockfalls into the lava lake trigger oscillations that are interpreted as acoustic-gravity wave eigenmodes of the system.

The governing equations are derived by considering small perturbations about a rest state of thermodynamic and mechanical equilibrium (the magmastatic base state); the liquid and gas phases are assumed to move with the same velocity. The exchange of mass between liquid and gas phases is described in terms of non-equilibrium bubble growth and resorption (BGR) of volatiles, parametrized as a relaxation process over a characteristic timescale $\tau$. The governing equations are obtained by linearizing the equations for mass and momentum balance in the magma, the BGR evolution equation involving the volatile solubility law, and the magma equation of state:

$$
\rho \frac{\partial v}{\partial t}+\frac{\partial p}{\partial z}+\frac{\rho g}{K} p-\rho g a n-\frac{\mu}{r} \frac{\partial}{\partial r}\left(r \frac{\partial v}{\partial r}\right)=0,
$$




$$
\begin{gathered}
\frac{1}{K} \frac{\partial p}{\partial t}+\frac{\partial u}{\partial z}-\frac{\rho g}{K} u+\frac{a(n+b p)}{\tau}=0 \\
\frac{\partial n}{\partial t}+\rho g b u+\frac{n+b p}{\tau}=0 \\
u=\frac{2}{R^{2}} \int_{0}^{R} v r d r
\end{gathered}
$$

where $p(t, z), v(t, z, r)$, and $u(t, z)$ are, as before, perturbations in pressure, velocity, and cross-sectionally averaged velocity; and $n(t, z)$ is the perturbation in the mass fraction of exsolved gas. In addition, $\rho(z)$ is the density in the magmastatic state, $K(z)$ is the bulk modulus, $g$ is acceleration due to gravity, $\mu$ is viscosity, $R$ is the conduit radius, and $a(z) \geq 0$ and $b(z) \geq 0$ are coefficients quantifying processes related to the influence of volatiles on the magma density and the pressure-dependence of volatile solubility. Note that these equations, unlike those in the model problem, are dimensional.

As in the model problem, we enforce a no-slip boundary condition:

$$
v(t, z, r=R)=0
$$

The top and bottom boundary conditions for the conduit require more careful treatment than in the model problem. At the bottom of the conduit $(z=0)$, choices of boundary condition include but are not limited to a no flow boundary $(v(t, z=0, r)=0)$ or a zero pressure perturbation boundary $(p(t, z=0)=0)$; we focus on the latter here. At the top of the conduit, the magma is in contact with the atmosphere, so pressure is set equal to atmospheric pressure (plus some perturbation $p_{\text {force }}(t)$ associated with the impulsive forcing from the rockfalls) on that moving surface located at $L+h(t)$, where $L$ is the nominal conduit length and $h(t)$ is the change in height. Linearization of this condition results in the top boundary condition

$$
p(t, z=L)-\rho(L) g h(t)=p_{\text {force }}(t)
$$

where the height evolves according to

$$
\frac{d h}{d t}=u(t, z=L) .
$$


Equations (6.2) and (6.3) are discretized using SBP operators on a regular grid with boundary conditions weakly enforced using simultaneous approximation terms (SAT) [14]. This discretization is possible to perform because there is no $r$ dependence in the variables $(p, n, u)$. The SAT technique is implemented using the method and choice of SAT parameters described in [3], where the target boundary values are determined by preserving the characteristic variables carrying information out of the domain. The radial diffusion term in (6.1) is discretized using the same operator $\mathbf{D}_{2}$ as in the model problem, i.e., by (4.5). The integral in (6.4) is discretized using the $\overline{\mathbf{P}}_{-}$operator, see (4.8) and (4.9). We note that viscosity was neglected in the original study [3]; accounting for viscous dissipation appears to be essential to properly match observations, thus motivating this study.

The simulation results for a conduit of length $L=2 \mathrm{~km}$ and radius $R=15 \mathrm{~m}$ containing magma with a viscosity of $\mu=10 \mathrm{~Pa}$ s are shown in Figures 7, 8, and 9. In these figures, the system is forced by a Gaussian pressure pulse $p_{\text {force }}(t)$ at the surface $(z=L)$ with an amplitude of $50 \mathrm{kPa}$ and $1 \mathrm{~s}$ duration. The increasing pressure with depth in the magmastatic base state, together with the pressure-dependence of volatile solubility, leads to segmentation of the conduit into two distinct sections. At depths below $z=L / 2$ (the exsolution depth), all volatiles are dissolved (making the magma less compressible and more dense); above the exsolution depth, gas bubbles are present, making the magma more compressible and less dense. As in the model problem (see Figure 3a), the viscous effects manifest in narrow boundary layers near the wall of the conduit and the amplitude of the oscillations decays with each cycle, as seen in Figure 7. 


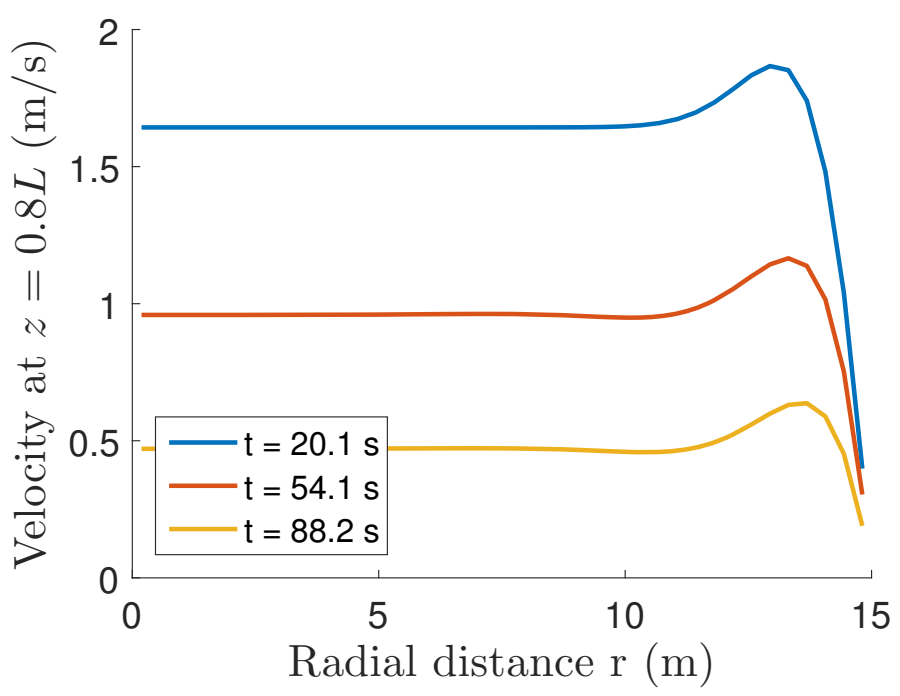

Figure 7: Radial profiles of the vertical magma velocity at $z=0.8 L$ (above the exsolution depth) for magma viscosity $\mu=10 \mathrm{~Pa}$ s. As in the model problem, the viscous effects manifest in boundary layers near the conduit wall.

The response of the magma column to the Gaussian pressure impulse (i.e., rockfall) is seen in Figure 8 and Figure 9, which show the time evolution of the pressure and fluid velocity at $r=R / 2$, respectively. The disturbance at the surface creates a wave that travels down into the conduit at a relatively constant speed until it reaches the exsolution depth, where it excites ringing behavior in the deeper conduit section. These oscillations are damped out by the fluid viscosity and non-equilibrium bubble growth and resorption over the course of several minutes. With the addition of viscosity, many features observed in the Kilauea data are reproduced in the model. 


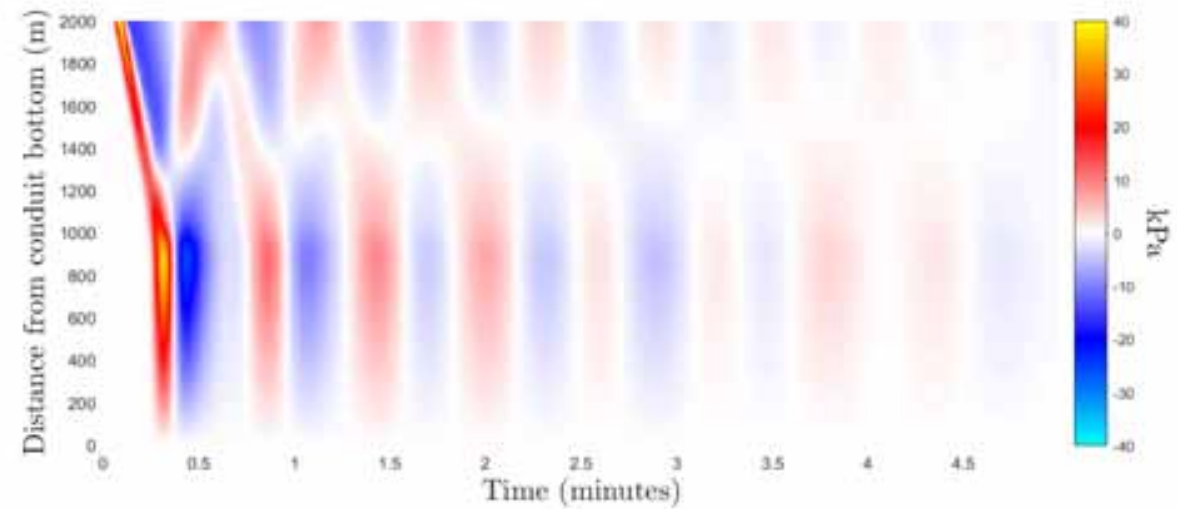

Figure 8: Space-time plot of pressure perturbation in response to rockfall onto the surface of the magma column.

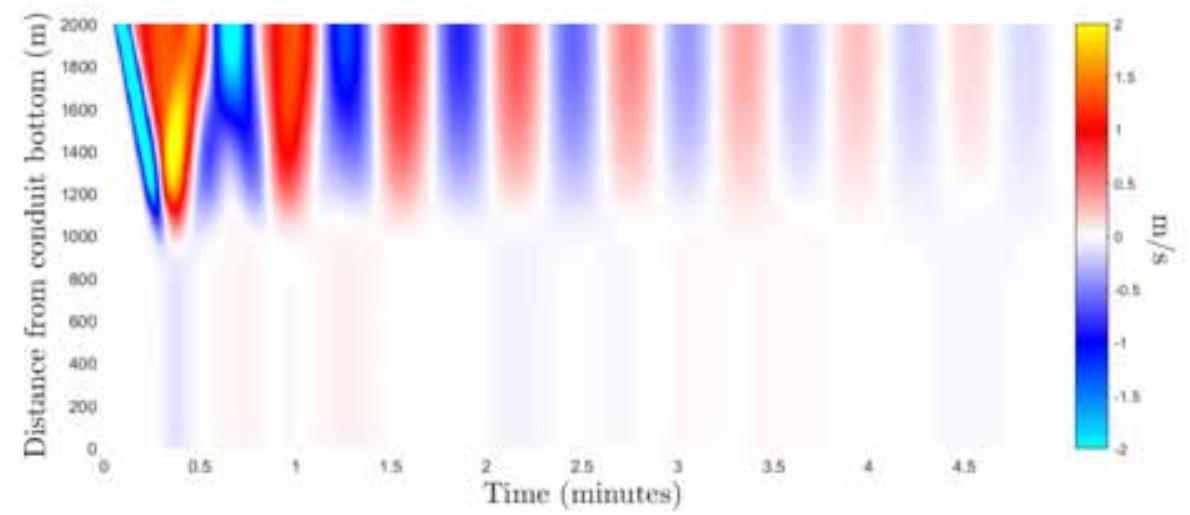

Figure 9: Space-time plot of vertical velocity along the vertical cross-section at $r=R / 2$ in response to rockfall onto the surface of the magma column.

\section{Conclusion}

We have developed a numerical model for the propagation of waves in a fluidfilled tube or conduit with dissipation due to fluid viscosity. To model viscous dissipation, we have developed a method for constructing a provably stable method introducing a summation-by-parts operator $\mathbf{D}_{2}$ for approximating the radial component of the Laplacian in cylindrical coordinates. This operator is 
constructed by defining a staggered grid in the radial direction, on which we define first derivative SBP difference operators. This operator does not include the grid points at the endpoints $r=0$ and $r=1$ and exactly enforces the no-slip boundary condition at $r=1$. To prove stability, we derived an energy estimate for the semi-discrete approximation.

As an example of an application problem which is not analytically solvable, we applied the numerical method to the governing equations for acousticgravity waves propagating in a magmatic conduit. This problem introduces complexities not seen in the model problem, including a multiphase fluid and depth-dependent material properties, which make it solvable only by numerical techniques. Related application problems arise in other contexts, including arterial flow [1] and human phonation [21], and could constitute a basis for further work.

A potential future development is to reduce the stencil width of the difference approximation. Since the approximation of the radial component of the Laplacian operator was constructed by applying the first derivative twice, this results in a stencil width of $4 p^{2}-1$, where $2 p$ is the interior accuracy. The optimal stencil width is $2 p+1$. For $p=1$ (second order interior accuracy), the stencil width is optimal. For higher orders of accuracy, it might be possible to construct a difference approximation of the Laplacian with optimal stencil width using the approaches taken in [22, 23, 24, 25].

While the analysis presented in this work is restricted to cylindrical coordinates, it is likely that the staggered grid operators could be used to handle similar coordinate singularities in spherical coordinates. Furthermore, our method of handling the $1 / r$ coordinate singularity does not make use of the symmetry condition at $r=0$ that is specific to axisymmetric problems, which indicates that this method could be applied to non-axisymmetric problems in both cylindrical and spherical coordinates. Extending this method to fully three dimensional non-axisymmetric problems would require the definition of derivative operators in the azimuthal direction, and careful treatment of the truncation step. 


\section{Acknowledgments}

This work was performed under the auspices of the U.S. Department of Energy by Lawrence Livermore National Laboratory under contract DE-AC5207NA27344 (technical report number LLNL-JRNL-698320).

\section{Funding}

This work was partially supported by a Chevron fellowship to O. O'Reilly.

\section{Appendix A. Accuracy of quadrature rules}

Lemma Appendix A.1. Let the $S B P$ operators $\mathbf{D}_{+}$and $\mathbf{D}_{-}$be pth-orderaccurate on the boundary. Then the order of the quadrature rules $\mathbf{P}_{+}$and $\mathbf{P}_{-}$ is at least $\max (2,(2 p-2))$ for $p \geq 1$.

Proof. Let $r_{j}=a+j h, h=(b-a) / N$, and $\mathbf{r}_{+}=\left[r_{0}, r_{1}, \ldots, r_{N}\right]^{T}$ be the vector of grid point locations for the regular grid. The corresponding location vector for the staggered grid is $\mathbf{r}_{-}=\left[r_{0}, r_{1 / 2}, \ldots, r_{N-1 / 2}, r_{N}\right]^{T}$. Denote a grid function on the regular grid by $\mathbf{u}_{+}=\left[u_{0}, u_{1}, \ldots, u_{N}\right]^{T}$ and on the staggered grid by $\mathbf{u}_{-}=\left[u_{0}, u_{1 / 2}, \ldots, u_{N-1 / 2}, u_{N}\right]^{T}$. Recall the summation-by-parts property (3.5) for first derivatives. By applying this property to the second derivatives $\mathbf{D}_{-} \mathbf{D}_{+}$and $\mathbf{D}_{+} \mathbf{D}_{-}$, and taking the scalar products (3.2) and (3.3) it follows that

$$
\begin{aligned}
& \left(\mathbf{u}_{+}, \mathbf{D}_{+} \mathbf{D}_{-} \mathbf{v}_{+}\right)_{+}=-\left(\mathbf{D}_{-} \mathbf{u}_{+}, \mathbf{D}_{-} \mathbf{v}_{+}\right)_{-}+u_{N}\left(\mathbf{D}_{-} \mathbf{v}_{+}\right)_{N}-u_{0}\left(\mathbf{D}_{-} \mathbf{v}_{+}\right)_{0} \\
& \left(\mathbf{u}_{-}, \mathbf{D}_{-} \mathbf{D}_{+} \mathbf{v}_{-}\right)_{-}=-\left(\mathbf{D}_{+} \mathbf{u}_{+}, \mathbf{D}_{+} \mathbf{v}_{-}\right)_{+}+u_{N}\left(\mathbf{D}_{+} \mathbf{v}_{-}\right)_{N}-u_{0}\left(\mathbf{D}_{-} \mathbf{v}_{-}\right)_{0}
\end{aligned}
$$

The SBP operators exactly satisfy

$$
\left\{\begin{array}{ll}
\mathbf{D}_{+} \mathbf{r}_{-}^{s} & =s \mathbf{r}_{+}^{s-1}, \\
\mathbf{D}_{-} \mathbf{r}_{+}^{s} & =s \mathbf{r}_{-}^{s-1}, \\
\mathbf{D}_{-} \mathbf{D}_{+} \mathbf{r}_{-}^{s} & =s(s-1) \mathbf{r}_{-}^{s-2}, \\
\mathbf{D}_{+} \mathbf{D}_{-} \mathbf{r}_{+}^{s} & =s(s-1) \mathbf{r}_{+}^{s-2},
\end{array} \quad s \in[1, p],\right.
$$

where $\mathbf{r}_{+}^{s}=\left[r_{0}^{s}, r_{1}^{s}, \ldots, r_{N}^{s}\right]^{T}$ and $\mathbf{r}_{-}^{s}=\left[r_{0}^{s}, r_{1 / 2}^{s}, \ldots, r_{N-1 / 2}^{s}, r_{N}^{s}\right]^{T}$. 
Case $\boldsymbol{I}: p=1$. In this case, $\mathbf{P}_{+}$is the trapezoidal rule in (3.7) and $\mathbf{P}_{-}$is the midpoint rule in (3.7), which are second-order-accurate.

Case II: Even degree polynomials. Taking $\mathbf{u}_{+}=\mathbf{r}_{+}^{s}, \mathbf{v}_{+}=\mathbf{r}_{+}^{s}, \mathbf{u}_{-}=\mathbf{r}_{-}^{s}$, and $\mathbf{v}_{-}=\mathbf{r}_{-}^{s}$ in (A.1) and (A.2), and using (A.3) yields

$$
\begin{array}{ll}
(s-1)\left(\mathbf{e}_{+}, \mathbf{r}_{+}^{2 s-2}\right)_{+}=-s\left(\mathbf{e}_{-}, \mathbf{r}_{-}^{2 s-2}\right)_{-}+r_{N}^{2 s-1}-r_{0}^{2 s-1}, & s \in[2, p], \\
(s-1)\left(\mathbf{e}_{-}, \mathbf{r}_{-}^{2 s-2}\right)_{-}=-s\left(\mathbf{e}_{+}, \mathbf{r}_{+}^{2 s-2}\right)_{+}+r_{N}^{2 s-1}-r_{0}^{2 s-1}, & s \in[2, p],
\end{array}
$$

where $\mathbf{e}_{+}=[1,1, \ldots, 1]^{T} \in \mathbb{R}^{N+1}$ and $\mathbf{e}_{-}=[1,1, \ldots, 1]^{T} \in \mathbb{R}^{N+2}$. By forming the difference between (A.5) and (A.4)

$$
\left(\mathbf{e}_{+}, \mathbf{r}_{+}^{2 s-2}\right)_{+}=\left(\mathbf{e}_{-}, \mathbf{r}_{-}^{2 s-2}\right)_{-} .
$$

Inserting (A.6) into (A.4) and (A.5) leads to

$$
\left(\mathbf{e}_{+}, \mathbf{r}_{+}^{2 s-2}\right)_{+}=\left(\mathbf{e}_{-}, \mathbf{r}_{-}^{2 s-2}\right)_{-}=\frac{1}{2 s-1}\left(b^{2 s-1}-a^{2 s-1}\right), \quad s \in[2, p] .
$$

The right-hand side is exactly equal to the result obtained by the integration

$$
\int_{a}^{b} r^{2 s-2} d r=\frac{1}{2 s-1}\left(b^{2 s-1}-a^{2 s-1}\right),
$$

which means that the quadrature rules are exact for all even degree polynomials up to degree $2 p-2$.

Case III: Odd degree polynomials. Now taking $\mathbf{u}_{+}=\mathbf{r}_{+}^{s}$ and $\mathbf{v}_{+}=\mathbf{r}_{+}^{s-1}$ and $\mathbf{u}_{-}=\mathbf{r}_{-}^{s}$ and $\mathbf{v}_{-}=\mathbf{r}_{-}^{s-1}$ in (A.1) and (A.2), and using (A.3) leads to

$$
\begin{array}{ll}
(s-2)\left(\mathbf{e}_{+}, \mathbf{r}_{+}^{2 s-3}\right)_{+}=-s\left(\mathbf{e}_{-}, \mathbf{r}_{-}^{2 s-3}\right)_{-}+r_{N}^{2 s-2}-r_{0}^{2 s-2}, & s \in[2, p], \\
(s-2)\left(\mathbf{e}_{-}, \mathbf{r}_{-}^{2 s-3}\right)_{-}=-s\left(\mathbf{e}_{+}, \mathbf{r}_{+}^{2 s-3}\right)_{+}+r_{N}^{2 s-2}-r_{0}^{2 s-2}, & s \in[2, p] .
\end{array}
$$

By forming the difference between (A.8) and (A.7)

$$
\left(\mathbf{e}_{+}, \mathbf{r}_{+}^{2 s-3}\right)_{+}=\left(\mathbf{e}_{-}, \mathbf{r}_{-}^{2 s-3}\right)_{-} .
$$

Inserting (A.9) into (A.7) and (A.8) leads to

$$
\left(\mathbf{e}_{+}, \mathbf{r}_{+}^{2 s-3}\right)_{+}=\left(\mathbf{e}_{-}, \mathbf{r}_{-}^{2 s-3}\right)_{-}=\frac{1}{2 s-2}\left(b^{2 s-2}-a^{2 s-2}\right), s \in[2, p],
$$


which means that the quadrature rules are exact for all odd degree polynomials up to degree $2 p-3$.

Remark 3. For $p>1$, there is numerical evidence that the quadrature rules are of order $2 p-1$.

\section{Appendix B. Proof of Proposition 4.1 (second order case)}

We need to show that the following holds:

$$
\overline{\mathbf{v}}^{T} \mathbf{A} \overline{\mathbf{v}}=\left\|\left(\mathbf{I}_{z} \otimes \overline{\mathbf{D}}_{+}\right) \overline{\mathbf{v}}\right\|_{\Omega_{+}}^{2}>0, \text { for all } \overline{\mathbf{v}} \neq 0
$$

i.e. $\mathbf{A}>0$. From the definition of the norm $\|\cdot\|_{\Omega_{+}}^{2}$ in (4.10), we get

$$
\mathbf{A}=\mathbf{P}_{z} \otimes\left(\overline{\mathbf{D}}_{+}^{T} \mathbf{P}_{+} \mathbf{R}_{+} \overline{\mathbf{D}}_{+}\right)
$$

Since $\mathbf{P}_{z}=\Delta z \mathbf{I}_{z}>0$, we only need to consider $\overline{\mathbf{A}}=\overline{\mathbf{D}}_{+}^{T} \mathbf{P}_{+} \mathbf{R}_{+} \overline{\mathbf{D}}_{+} \in \mathbb{R}^{N \times N}$. If $\overline{\mathbf{A}}>0$ then the null space $\operatorname{null}(\overline{\mathbf{A}})=\left\{\mathbf{x} \in \mathbb{R}^{N} \neq 0 \mid \mathbf{A x}=0\right\}$ is empty. Since $\mathbf{R}_{+}$is zero on the diagonal in the first row and both $\mathbf{P}_{+}$and $\mathbf{R}_{+}$are diagonal and positive everywhere else, it is sufficient to show that there exists no vector $\overline{\mathbf{x}}=\left(\begin{array}{lllll}\bar{x}_{0} & \bar{x}_{1} & \ldots & \bar{x}_{N-1}\end{array}\right)^{T} \in \mathbb{R}^{N} \neq 0$ such that $\overline{\mathbf{D}}_{+} \overline{\mathbf{x}}=\mathbf{y}$, where $\mathbf{y}=(\alpha, 0,0, \ldots 0)^{T} \in \mathbb{R}^{(N+1)}$ for $\alpha \neq 0$. In the second order case, the matrix $\overline{\mathbf{D}}_{+}$is given by

$$
\overline{\mathbf{D}}_{+}=\frac{1}{\Delta r}\left[\begin{array}{cccccc}
2 & & & & & \\
-1 & 1 & & & & \\
& -1 & 1 & & & \\
& & -1 & 1 & & \\
& & & \ddots & \ddots & \\
& & & & -1 & 1 \\
& & & & & -2
\end{array}\right] \in \mathbb{R}^{(N+1) \times N} .
$$

Consider the equation system $\overline{\mathbf{D}}_{+} \overline{\mathbf{x}}=0$. We use a proof by contradiction and assume that there exists $\mathbf{x} \neq 0$. From the last equation we obtain $\bar{x}_{N-1}=0$. 


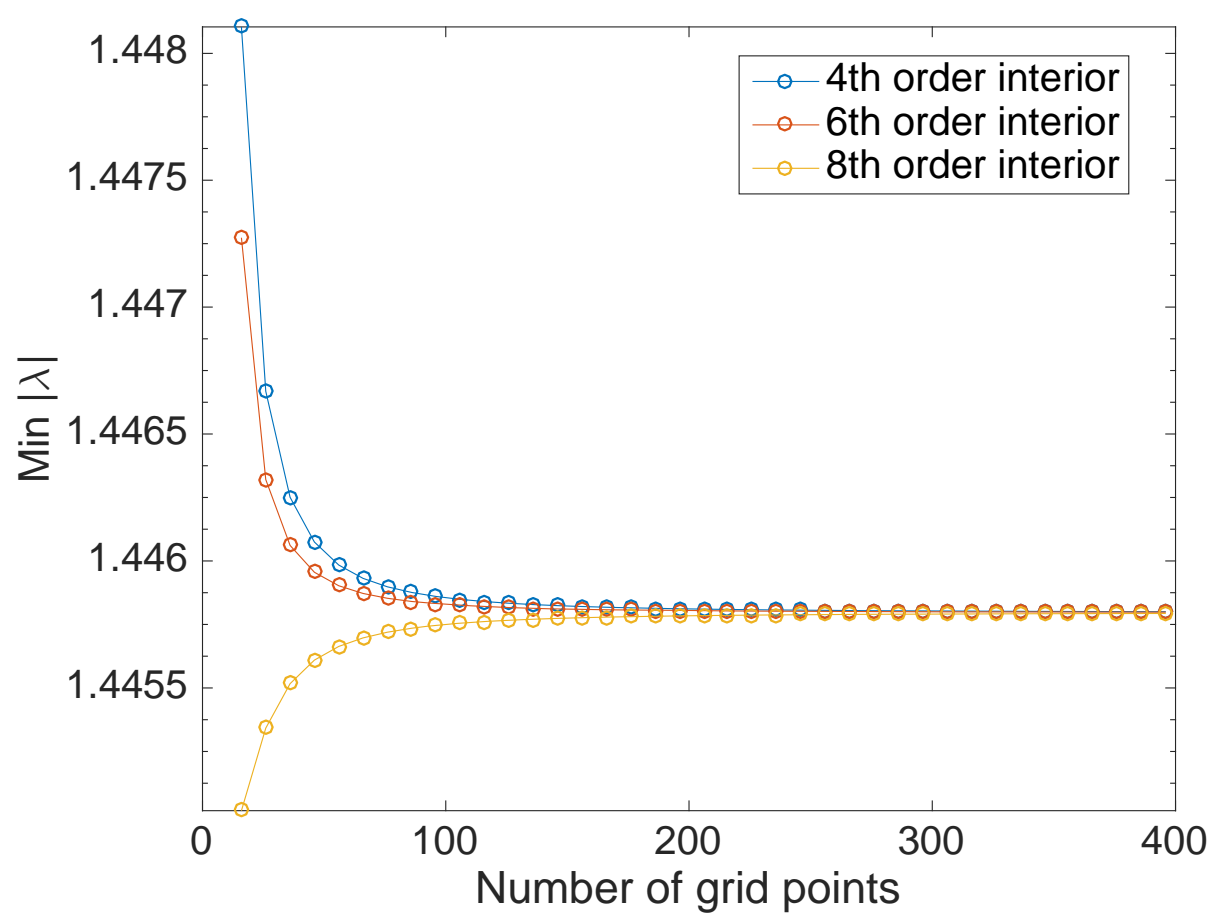

Figure B.10: The minimum modulus eigenvalue of $\overline{\mathbf{A}} / \Delta r$ as a function of the number of grid points.

Inserting this result into the second last equation yields $\bar{x}_{N-2}=0$. Repeating the procedure until the second equation yields $\bar{x}_{0}=0$, which implies that $\overline{\mathbf{x}}=0$ is the only solution. We have arrived at a contradiction and therefore conclude that $\overline{\mathbf{A}}>0$, which completes the proof.

For higher order of accuracy, we numerically compute the minimum modulus eigenvalue

$$
\lambda_{\min } \equiv \min _{\lambda_{i} \in \lambda(\overline{\mathbf{A}} / \Delta r)}\left|\lambda_{i}\right| .
$$

We use 16 to 400 grid points. Figure B.10 shows that $\lambda_{\min }$ is nonzero.

\section{References}

[1] J. R. Womersley, Method for the calculation of velocity, rate of flow and viscous drag in arteries when the pressure gradient is known, The Jour- 
nal of Physiology 127 (3) (1955) 553-563. doi:10.1113/jphysiol.1955. sp005276.

URL http://dx.doi.org/10.1113/jphysiol.1955.sp005276

[2] H. B. Atabek, H. S. Lew, Wave propagation through a viscous incompressible fluid contained in an initially stressed elastic tube, Biophys J 6 (4) (1966) 481-503, 19210972[pmid].

URL http://www.ncbi.nlm.nih.gov/pmc/articles/PMC1367963/

[3] L. Karlstrom, E. M. Dunham, Excitation and resonance of acoustic-gravity waves in a column of stratified, bubbly magma, Journal of Fluid Mechanics 797 (2016) 431-470. doi:10.1017/jfm.2016. 257.

URL http://journals.cambridge.org/article_S0022112016002573

[4] E. Tavakoli, B. Lessani, R. Hosseini, High-order pole-treatment in cylindrical coordinates for incompressible flow simulations with finite-difference collocated schemes, Journal of Computational Physics 296 (2015) 1 - 24. doi:http://dx.doi.org/10.1016/j.jcp.2015.04.042.

URL http://www.sciencedirect.com/science/article/pii/ S0021999115002995

[5] R. Oguic, S. Viazzo, S. Poncet, A parallelized multidomain compact solver for incompressible turbulent flows in cylindrical geometries, Journal of Computational Physics 300 (2015) 710 - 731. doi:http://dx.doi.org/10.1016/j.jcp.2015.08.003.

URL http://www.sciencedirect.com/science/article/pii/ S0021999115005203

[6] M. D. Griffin, E. Jones, J. D. Anderson, A computational fluid dynamic technique valid at the centerline for non-axisymmetric problems in cylindrical coordinates, Journal of Computational Physics 30 (3) (1979) 352 360. doi:http://dx.doi.org/10.1016/0021-9991(79)90120-7.

URL http://www.sciencedirect.com/science/article/pii/ 0021999179901207 
[7] G. Constantinescu, S. Lele, A highly accurate technique for the treatment of flow equations at the polar axis in cylindrical coordinates using series expansions, Journal of Computational Physics 183 (1) (2002) 165 - 186. doi:http://dx.doi.org/10.1006/jcph.2002.7187.

URL http://www.sciencedirect.com/science/article/pii/ S0021999102971871

[8] Y. Morinishi, O. V. Vasilyev, T. Ogi, Fully conservative finite difference scheme in cylindrical coordinates for incompressible flow simulations, Journal of Computational Physics 197 (2) (2004) 686 - 710. doi:http://dx.doi.org/10.1016/j.jcp.2003.12.015.

URL http://www.sciencedirect.com/science/article/pii/ S0021999103006594

[9] K. Mohseni, T. Colonius, Numerical treatment of polar coordinate singularities, Journal of Computational Physics 157 (2) (2000) 787 - 795. doi:http://dx.doi.org/10.1006/jcph.1999.6382.

URL http://www.sciencedirect.com/science/article/pii/ S0021999199963829

[10] R. Verzicco, P. Orlandi, A finite-difference scheme for threedimensional incompressible flows in cylindrical coordinates, Journal of Computational Physics 123 (2) (1996) 402 - 414. doi:http: //dx.doi.org/10.1006/jcph.1996.0033.

URL http://www.sciencedirect.com/science/article/pii/ S0021999196900339

[11] H.-O. Kreiss, G. Scherer, Finite element and finite difference methods for hyperbolic partial differential equations, in: C. d. Boor (Ed.), Mathematical Aspects of Finite Elements in Partial Differential Equations, Academic Press, 1974, pp. 195 - 212. doi:http://dx.doi.org/10.1016/B978-0-12-208350-1.50012-1. 
URL

http://www.sciencedirect.com/science/article/pii/

B9780122083501500121

[12] B. Strand, Summation by parts for finite difference approximations for d/dx, Journal of Computational Physics 110 (1) (1994) 47 - 67. doi:http://dx.doi.org/10.1006/jcph.1994.1005.

URL http://www.sciencedirect.com/science/article/pii/ S0021999184710059

[13] M. Svärd, J. Nordström, Review of summation-by-parts schemes for initial-boundary-value problems, Journal of Computational Physics 268 (2014) 17 - 38. doi:http://dx.doi.org/10.1016/j.jcp.2014.02.031.

URL http://www.sciencedirect.com/science/article/pii/ S002199911400151X

[14] M. H. Carpenter, D. Gottlieb, S. Abarbanel, Time-stable boundary conditions for finite-difference schemes solving hyperbolic systems: Methodology and application to high-order compact schemes, Journal of Computational Physics 111 (2) (1994) $220 \quad$ - 236. doi:http://dx.doi.org/10.1006/jcph.1994.1057.

URL http://www.sciencedirect.com/science/article/pii/ S0021999184710576

[15] D. C. D. R. Fernández, J. E. Hicken, D. W. Zingg, Review of summation-byparts operators with simultaneous approximation terms for the numerical solution of partial differential equations, Computers \& Fluids 95 (2014) 171 - 196. doi:http://dx.doi.org/10.1016/j.compfluid.2014.02.016.

URL http://www.sciencedirect.com/science/article/pii/ S0045793014000796

[16] G. Calabrese, D. Neilsen, Spherical excision for moving black holes and summation by parts for axisymmetric systems, Phys. Rev. D 69 (2004) 044020. doi:10.1103/PhysRevD.69.044020.

URL http://link.aps.org/doi/10.1103/PhysRevD.69.044020 
[17] C. Gundlach, J. M. Martín-García, D. Garfinkle, Summation by parts methods for spherical harmonic decompositions of the wave equation in any dimensions, Classical and Quantum Gravity 30 (14) (2013) 145003.

URL http://stacks.iop.org/0264-9381/30/i=14/a=145003

[18] M. H. Carpenter, C. A. Kennedy, Fourth-order 2n-storage runge-kutta schemes, Tech. Rep. NASA-TM-109112, NASA (June 1994).

URL http://ntrs. nasa.gov/search. jsp?R=19940028444

[19] B. Gustafsson, The convergence rate for difference approximations to mixed initial boundary value problems, Mathematics of Computation 29 (130) (1975) 396-406.

URL http://www.jstor.org/stable/2005559

[20] M. Svärd, J. Nordström, On the order of accuracy for difference approximations of initial-boundary value problems, Journal of Computational Physics 218 (1) (2006) 333-352. doi:10.1016/j.jcp.2006.02.014.

URL http://dx.doi.org/10.1016/j.jcp.2006.02.014

[21] M. Larsson, B. Müller, Numerical simulation of confined pulsating jets in human phonation, Computers \& Fluids 38 (7) (2009) 1375 - 1383, special Issue Dedicated to Professor Alain Lerat on the Occasion of his 60th Birthday. doi:http://dx.doi.org/10.1016/j.compfluid.2008.01.033.

URL http://www.sciencedirect.com/science/article/pii/ S0045793008000662

[22] S. Nilsson, N. A. Petersson, B. Sjögreen, H.-O. Kreiss, Stable difference approximations for the elastic wave equation in second order formulation, SIAM Journal on Numerical Analysis 45 (5) (2007) 1902-1936. doi:10. $1137 / 060663520$.

URL http://dx.doi.org/10.1137/060663520

[23] K. Mattsson, Summation by parts operators for finite difference approximations of second-derivatives with variable coefficients, Journal of Scientific 
Computing 51 (3) (2012) 650-682. doi:10.1007/s10915-011-9525-z.

URL http://dx.doi.org/10.1007/s10915-011-9525-z

[24] B. Sjögreen, N. A. Petersson, A fourth order accurate finite difference scheme for the elastic wave equation in second order formulation, Journal of Scientific Computing 52 (1) (2012) 17-48. doi:10.1007/ s10915-011-9531-1.

URL http://dx.doi.org/10.1007/s10915-011-9531-1

[25] D. C. D. R. Fernández, D. W. Zingg, Generalized summation-by-parts operators for the second derivative, SIAM Journal on Scientific Computing 37 (6) (2015) A2840-A2864. arXiv:http://dx.doi.org/10.1137/ 140992205, doi:10.1137/140992205.

URL http://dx.doi.org/10.1137/140992205 